\title{
Revealed Political Power
}

\section{Jinhui H. Bai ${ }^{*}$ and Roger Lagunoff ${ }^{\dagger}$}

June, $2012^{\ddagger}$

\begin{abstract}
This paper examines the problem of inferring bias in a political system. We consider the view of an "outside observer" who sees a finite sequence of policy data, but observes neither the citizens' preference profile nor the underlying distribution of political power. He views political power as if it were derived from a wealth-weighted voting system, where the weights determine the nature and magnitude of the wealth bias. Positive weights on relative income favors the rich while negative ones favor the poor. A preparatory result establishes that any policy data may be rationalized by any system of wealth-weighted voting. However, when the observations include polling data, we show that policy and polling observations together imply explicit upper and lower bounds on the set of rationalizing biases. Over time, accumulated data narrows this band, hence sharpens the observer's inference. Moreover, natural restrictions on preferences rule out the unbiased case of equal representation. The inferential model is shown to be consistent with a simple model of political competition with endogenous campaign contributions.
\end{abstract}

JEL Codes: C73, D63, D72, D74, H11

Key Words and Phrases: wealth-bias, polling data, weighted majority winner, rationalizing weights, Universal Bias Principle.

\footnotetext{
*Department of Economics, Georgetown University, Washington, DC 20057, USA. +1-202-687-0935, jb543@georgetown.edu, https://sites.google.com/site/jinhuibai/.

${ }^{\dagger}$ Department of Economics, Georgetown University, Washington, DC 20057, USA. +1-202-687-1510, lagunofr@georgetown. edu, www.georgetown.edu/lagunoff/lagunoff.htm.

${ }^{\ddagger}$ We thank seminar participants at Cal Tech, Indiana, Maryland, Northwestern, Stanford, Towson, UC Santa Barbara, Wisconsin, Warwick, The PIER (Penn) Conference, the SED Conference, the Summer Econometric Society Meetings, the Stanford-Kingston Political Economy Conference, and the Midwest Theory Conference, the Summer NBER, the Paris Dauphine Workshop on Economic Theory, the LSU Bargaining Conference, and especially Roland Bénabou for helpful comments and suggestions on an earlier draft of this paper. We also thank Jan Eeckout and three anonymous referees for their many helpful recommendations.
} 


\section{Introduction}

On paper, electoral processes in most democracies satisfy some rough form of political egalitarianism, often taking the form of "one-man-one-vote" electoral systems. ${ }^{1}$ It is unlikely, however, that the de facto distribution of power in these countries is equal. There is anecdotal evidence, and some systematic evidence, that wealth matters in the political process.

Rosenstone and Hansen (1993), for instance, show that the propensity to participate in every reported form of political activity rises with income. Campante (2011) uses campaign contribution data in the 2000 US presidential election to show that increases in income inequality raises the share of contributions coming from relatively wealthy individuals. Bartels (2008) offers a sweeping look at the relation between economic and political inequality. He examines whether economic inequality creates political inequality in the policy process. Using data from the Senate Election Study, he finds that Senators' voting records are unresponsive to the preferences of those in the lower third of the income distribution. ${ }^{2}$

These studies all suggest some form of wealth-bias in the political system. They find that the de facto allocation of power is such that richer individuals have a disproportionate influence in the policy process. The result is that policies enacted appear to favor wealthier rather than poorer individuals.

The present paper takes a step back by asking whether and how bias can be inferred directly from policies. When, for instance, can the egalitarian distribution of power based on "one-man-one-vote" be ruled out?

To address these issues we model an economy and its political system from the point of view of an "outside observer." The observer observes both policies and income distribution at finitely many dates. He does not observe, however, the underlying preference profile of the citizens whose political choices determined the policies. Instead, the observer knows only that policy preferences are differentiated by income. Richer individuals view policies differently than poorer ones. The observer's task is to infer something about the underlying distribution of political power that generated the observed policies.

To make sense of this inference problem, a "detail-free" model of political power is formulated as follows. Consider a political system that produces policies as if they resulted from a weighted majority voting process in which an individual's vote share depended on his income. This implied vote share rule depends, in turn, on an unobserved parameter - the "wealth bias." The wealth bias determines how the individual's income affects his vote share. When

\footnotetext{
${ }^{1}$ Examples include Winner-take-all Presidential elections (in the U.S. and Latin America) and Proportional Representation in Parliamentary elections (e.g., Western Europe). There are also a few well known exceptions, including the U.S. Senate in which representation is equal across states, so that voters in small states have disproportionate political power.

${ }^{2}$ By contrast, Senators' responsiveness to the middle and upper thirds is shown to be virtually linear to income. See Chapter 9 of Bartels (2008).
} 
the bias is positive, one's vote share increases with one's income; when the bias is negative, vote share is decreasing in income. When its value is zero (the unbiased case), vote share is constant across all income levels.

We characterize such vote share rules axiomatically. A canonical special case is found in Bénabou (1996, 2000). There, the vote share rule arises from differential voting participation between rich and poor. The wealth bias there conforms to an income elasticity for voting participation. Another example, shown in the paper, arises from differences in campaign contributions, with the bias determining the marginal productivity of a contribution.

In all cases, if the bias were fully known, then one could relate income inequality to political inequality by calculating a "Political Lorenz Curve" which gives the implied vote share (hence, "political power") of the poorest $j$ th portion of the population, for each $j$. Hence, the observer must infer something about the wealth bias. The observer asks: what are the biases that are consistent with vote share rules that rationalize the observed policies as Weighted majority winners $(W M W)$ under an admissible preference profile?

A preparatory result establishes that, without further structure on preferences and/or data, any policy data can be rationalized by any wealth bias. In other words, in the benchmark case, policy data alone are not very discerning; it is consistent with any level of bias.

In order to develop a meaningful theory of inference, we therefore take two independent routes. First, we allow the observer to access polling data. Polls provide aggregate comparisons between various policies under consideration in the political process. A variety of common polling formats are considered, each pitting the observed policy against an arbitrary number of alternatives. Second, we further restrict the admissible preference domain.

To see why polling data helps, consider a poll at some date $t$ that pits the observed policy against a "right-wing" alternative (i.e., an alternative located to the right of the observed policy). Suppose that the poll reveals that portion $p_{t}$ of the population prefer the observed policy to the alternative. Under single crossing, these individuals belong to the poorest $p_{t}$ portion of the population. Yet, the fact that the observed policy must have resulted from a weighted voting process tells us that the wealthiest $1-p_{t}$ portion of the population must have had a weighted vote share smaller than $50 \%$. If this were not the case, then the richest group would have had the clout to veto the observed policy in favor of the alternative. Consequently, the income weights can be no greater than that necessary to lift the $1-p_{t}$ wealthiest individuals up to the $50 \%$ weighted voting threshold. This in turn defines an upper bound for the wealth bias - the largest possible bias in favor of wealthy individuals. Similarly, a poll that compares the observed policy to a "left-wing" alternative can be used to infer a lower bound. Both bounds can be explicitly calculated in each state of the economy.

More generally, the paper's main results establish upper and lower bounds on the set of wealth biases that jointly rationalize both the policy and poll data. We show that these bounds on the wealth bias are invariant to any of the common polling formats. Moreover, 
the bounds shrink as the data accumulate over time, allowing the observer to make a more precise prediction in the long run.

As for restrictions on the preference domain, two varieties are considered. One is a single crossing restriction on policy as the state varies. This preference restriction is natural in a growing economy with very minor distributional change over time. The other is a separability condition on policy and income. Separability is shown to arise naturally in economies with distributional changes but no growth. Each can be shown to rule out the unbiased polity when the data vary non-monotonically.

Overall, the inferential model is framed in a way that avoids specific parametric assumptions on preferences and sidesteps specific causal theories for why and how wealth bias might arise. In this sense, our work parallels a strain of Revealed Preference Theory (RPT) which examines whether finite consumption data is consistent with budget-constrained utility maximization. ${ }^{3}$ However, as a useful check, we show that the inferential model is consistent with a simple parametric model of political competition with endogenous voting and campaign contribution decisions. The bias then arises from endogenous differentials in contributions between rich and poor.

The paper is organized as follows. Section 2 lays out the basic model and formulates an implied voting process with latent wealth-weights. There, the notion of a rationalizing bias is introduced, and a benchmark result is described. Section 3 introduces poll data and uses that data to derive restrictions on the implied bias. We use these restrictions to examine the link between economic and political inequality. Section 4 examines inference under a more restricted preference domain. Section 5 demonstrates consistency of the inferential model with a parametric model of campaign contributions. Section 6 concludes with a discussion of the related literature. An Appendix follows with the proofs.

\section{The Model}

This section formulates an inferential model from the point of view of an outside observer. The tangible attributes of the economy such as income distribution and policies are observable. The observer does not see either the parametric preferences, or the underlying power distribution that produced the observed policies. Both the observed and unobserved attributes are laid out as follows.

\footnotetext{
${ }^{3}$ The classic reference is Afriat (1967). See also Richter (1966) and more recently Varian (2006) for summaries and surveys of RPT developed by Paul Samuelson and others.
} 


\subsection{What the Observer sees}

There are $T<\infty$ observation dates that give the outside observer a "window" into an ongoing economy. At each observation date $t=1, \ldots, T$, the observer observes a policy $a_{t}$ and an aggregate state variable $\omega_{t}$. The policy could be a tax rate, a public good, or some defined level of redistribution, and is determined by a political process (to be described shortly). The state may be an economy-wide public capital stock, such as public infrastructure. However, it could also represent a summary statistic of ideological characteristics of voters. Formally, $a_{t} \in A$ with $A$ a compact interval in $\mathbb{R}$, and $\omega_{t} \in \Omega$ where $\Omega$ is a connected subset of $\mathbb{R}$. Each state $\omega_{t}, t=1, \ldots, T$ viewed by the observer is assumed to be distinct. Subsequent references to the "data" will be taken to mean the observed sequence $\left\{a_{t}, \omega_{t}\right\}_{t=1}^{T}$.

The economy is populated by a continuum $I=[0,1]$ of citizen-types. A citizen-type is an index that orders individuals by income, with higher types accorded higher income. A citizen of type $i \in I$ holds income $y\left(i, \omega_{t}\right)$ in period $t$ that depends, potentially, on the value of the state $\omega_{t}$. The function $y$ is assumed to be continuous and increasing in $i$, with $y\left(0, \omega_{t}\right)>0 .{ }^{4}$ The monotonicity of $y$ in $i$ means that higher citizen types are wealthier, state-by-state. The assumption also implies a well defined conditional distribution function $i=F\left(\tilde{y} ; \omega_{t}\right)$ corresponding to the proportion of types holding income no greater than $\tilde{y}$ given the state $\omega_{t}$. The function $y$ is assumed to be known or viewed by the observer.

In the subsequent notation, $\omega_{t}$ and $a_{t}$ will refer to the on-path observations at date $t$, while $\omega$ and $a$ will connote a generic state and policy, resp., either on the observed path or off it. At this point, the model can be viewed in either of two ways.

1. The "classic" Revealed Preference Theory (RPT) interpretation. Here, the observer sees $\left\{a_{t}, \omega_{t}\right\}_{t=1}^{T}$ and presumes no intertemporal connection between observations. This is either because the data represent different replica economies, or because the data constitute a time series generated by myopic citizens.

2. The Dynamic Economy interpretation. As before the observer sees $\left\{a_{t}, \omega_{t}\right\}_{t=1}^{T}$. This time, however, he infers an intertemporal connection and can, in fact, back out a transition rule $\omega_{t+1}=Q\left(\omega_{t}, a_{t}\right)$ (on path) from the data. Here, the underlying time horizon may be infinite, and policy choices are determined by the aggregated decisions of a forward looking citizenry.

The first (static) interpretation is easier to describe in concrete terms, and so the examples and discussion are cast in terms of Interpretation \#1. However, all the results apply to either interpretation. ${ }^{5}$

\footnotetext{
${ }^{4}$ From here on, the term "increasing" will be taken to mean "strictly increasing", and the term "weakly increasing" will be taken to mean "nondecreasing".

${ }^{5}$ The Appendix contains a fuller elaboration of Interpretation \#2 and connects it to our main results.
} 


\subsection{What the Observer does not see}

Critically there are certain features of the economy that the observer cannot see. The observer does not observe the precise form of payoffs. A citizen of type $i$ has preferences over policy choices in $A$ expressed by a payoff function $U(i, \omega, a)$. The observer knows only that $U$ belongs to a set of admissible payoff functions satisfying two properties:

(A1) (Single Peakedness) $U$ is continuous in the index $i$, and single peaked in $a$.

(A2) (Single Crossing) $U$ satisfies the strict single crossing property in $(a ; i) .{ }^{6}$

The strict single crossing property (A2) implies that in every state, wealthier citizens always prefer larger policies than poorer citizens. The "strictness" is assumed to avoid the trivial case in which all individuals' tastes are identical. The single crossing assumption is critical for addressing the question of wealth bias. Without it, preferences may no longer be ordered by wealth, and so statements about certain policies "favoring richer" or "favoring poorer" individuals are no longer meaningful. ${ }^{7}$

Any payoff function $U$ satisfying (A1)-(A2) is referred to as an admissible preference profile. Note that the "restrictiveness" of this class of profiles strengthens rather than weakens some of our results. The larger the set of admissible preference orderings, the easier it is to find one that "works" in the sense that a political system can produce the policy data under such preferences. The narrower the preference class the more difficult it is to generate the data.

In addition to preferences, certain features of the political system remain hidden to our observer. Our observer approaches the inference problem by viewing political power as if it were derived from an explicit system of income-weighted vote shares with each policy outcome determined by pairwise voting. Formally, a vote share rule is a function $\lambda$ that determines an income weighted vote share $\lambda(y(i, \omega)$; $\alpha)$ for citizen-type $i$, where $\lambda$ is a continuous, integrable function of individual income $y(i, \omega)$ and a parameter $\alpha \in \mathbb{R}$. The parameter $\alpha$ determines how much weight is given to income or wealth such that the observed policy would be a majority winning outcome under a voting scheme with vote share $\lambda(y(i, \omega) ; \alpha)$ for type $i$. Larger values of $\alpha$ correspond to greater political weight accorded to the rich. Accordingly we refer to $\alpha$ as the bias of the rule.

\footnotetext{
${ }^{6} \mathrm{~A}$ function $f(x, y)$ is said to satisfy the single crossing property in $(x ; y)$ if for all $x>\hat{x}$ and $y>\hat{y}$, $f(x, \hat{y})-f(\hat{x}, \hat{y})(>) \geq 0$ implies $f(x, y)-f(\hat{x}, y)(>) \geq 0$, and satisfies strict single crossing in $(x ; y)$ if $f(x, \hat{y})-f(\hat{x}, \hat{y}) \geq 0$ implies $f(x, y)-f(\hat{x}, y)>0$. The "single crossing" as described here may be more accurately described as "single crossing from below." But because policies have no specific interpretation, notions of "larger" and "smaller" are arbitrary. Hence, without loss of generality, we could also have assumed single crossing from above.

${ }^{7}$ Clearly, single crossing is unnatural in some interesting environments, including those with religious and/or ideological cleavages, or in cases in which the issue allies the rich and poor together against the middle class.
} 
By formulating the inference problem in this way, we do not pin the source of wealth bias to a particular causal theory. There are, in any case, a number of theories that are consistent with the present formulation. Examples include:

- Endogenous turnout: $\alpha$ is an unobserved cost parameter that determines the effect of income $y(i, \omega)$ on turnout rate $\lambda(y(i, \omega) ; \alpha)$ for citizen $i$.

- Campaign contributions: $\alpha$ is an unobserved technological parameter determining the productivity of a contribution which, in turn, determines $i$ 's effective contribution $\lambda(y(i, \omega) ; \alpha)$.

- Valence: $\alpha$ determines the effect of income on ideological preference for determining the likelihood $\lambda(y(i, \omega) ; \alpha)$ that citizen $i$ votes for the more right-leaning candidate.

Implicit in these examples is an assumption we maintain throughout the paper that the outside observer knows the functional form of $\lambda$ but does not observe parameter $\alpha$. This isolates the latent parameter $\alpha$ as the object of interest. To illustrated the efficacy of this approach, we later develop one of these examples - a simple causal theory of bias based on campaign contributions - formally, and show that $\lambda$ may be interpreted concretely from the theory. For now, we illustrate how the observer can make effective inference in an example below.

\subsection{A Canonical Example}

This section describes a concrete application of the inferential model. We give explicit functional forms for both the vote share rule and the preference profile in an economy with a tax-supported public good. We then describe how the observer might approach the inference problem in this case.

Consider first the canonical vote share rule given by exponential weighting used by Bénabou (2000) in his study of inequality and its effects on redistribution:

$$
\lambda(\tilde{y} ; \alpha)=\tilde{y}^{\alpha}
$$

In this case $\alpha$ exponentially weights wealth. One can then interpret $1-\alpha$ as the weight attached to equal vote share or equal representation in voting. This interpretation is somewhat misleading, however, since the $\alpha$ can take values in the entire real line. Nevertheless, $\alpha$ may be directly interpreted as an income elasticity of vote share. When, for instance, $\alpha=1$ then a $10 \%$ increase in one's income leads to a $10 \%$ increase in his vote share, hence his political power.

Notice here that political power (as determined by vote share) is increasing in income if $\alpha>0$, decreasing if $\alpha<0$, and invariant to income if $\alpha=0$. Hence, the value $\alpha$ can be thought of as a measure of the extent of wealth bias. When $\alpha=0$, the political system may 
be said to be unbiased in the sense that each person's political weight in the distribution is invariant to income, hence all individuals are political equals. Following, for instance, Bénabou (1996), we will refer to $\alpha>0$ as the case of an elitist bias since wealth is rewarded in the political system; the case of $\alpha<0$ is referred to as a populist bias since political power is redistributed away from wealth. The cases where $|\alpha|>1$ are particularly stark in this example since this indicates a distribution of power that disproportionately rewards the fringes of the distribution. Extreme inequality occurs in the limit as $|\alpha| \rightarrow \infty$.

From the vote share rule one can define the distribution

$$
L^{p}(j ; \alpha, \omega) \equiv \frac{\int_{0}^{j} \lambda(y(i, \omega) ; \alpha) d i}{\int_{0}^{1} \lambda(y(i, \omega) ; \alpha) d i}=\frac{\int_{0}^{j} y(i, \omega)^{\alpha} d i}{\int_{0}^{1} y(i, \omega)^{\alpha} d i}
$$

We refer to $L^{P}$ as the Political Lorenz Curve since it gives a simple measure of political inequality. It describes the proportion of political power held by the poorest fraction $j$ of types in state $\omega$. Political inequality, as measured by $L^{P}$, can then change over time due to changes in the income distribution.

Figure 1a displays the two Lorenz curves in the case where the Political Lorenz Curve exhibits a "dampened" elitist bias. Specifically, $0<\alpha<1$, meaning that wealthier individuals have greater political weight than do poorer individuals, however, their increased weight is smaller than their weight in the income distribution. Political inequality therefore lies somewhere between income inequality and full equality. Figure $1 \mathrm{~b}$ displays the Political Lorenz curve when $\alpha>1$. In that case the elitist bias is more pronounced, with political inequality that exceeds income inequality in the degree that the wealthy are accorded power. Note that the two curves coincide in the case where $\alpha=1$. Figure 2 illustrates the case of a populist bias, i.e., $\alpha<0$. Most theories we are aware of predict an elitist bias if any. Nevertheless, it does not seem sensible to rule out the $\alpha<0$ case, a priori.

Now suppose the observer sees a policy outcome, knowing that the vote share rule is given by (1). The rule is applied to a stylized model of public goods with preferences given by,

$$
u\left(c_{t}, G_{t}\right)=c_{t}+\frac{G_{t}^{1-\rho}}{1-\rho} \quad \rho \in[0,1)
$$

where $G_{t}=\tau_{t} \int_{0}^{1} y\left(i, \omega_{t}\right) d i=\tau_{t} \bar{y}\left(\omega_{t}\right)$ is a collective good funded by a flat tax $\tau_{t}$, and $c_{t}=$ $\left(1-\tau_{t}\right) y\left(i, \omega_{t}\right)$ is after-tax private consumption. Though this is framed as a problem of public good provision, notice that if $\rho=0$, the problem reduces to one of pure redistribution. In terms of the present model, we define $a_{t}=1-\tau_{t}$ so the problem becomes

$$
U\left(i, \omega_{t}, a_{t}\right)=a_{t} y\left(i, \omega_{t}\right)+\frac{\left[\left(1-a_{t}\right) \bar{y}\left(\omega_{t}\right)\right]^{1-\rho}}{1-\rho} .
$$

The payoff function $U$ depends on $\omega_{t}$, not only through income $y(i, \omega)$, but also through $\bar{y}\left(\omega_{t}\right)$. It is easy to verify that this economy is consistent with the model (under Interpretation \#1) 


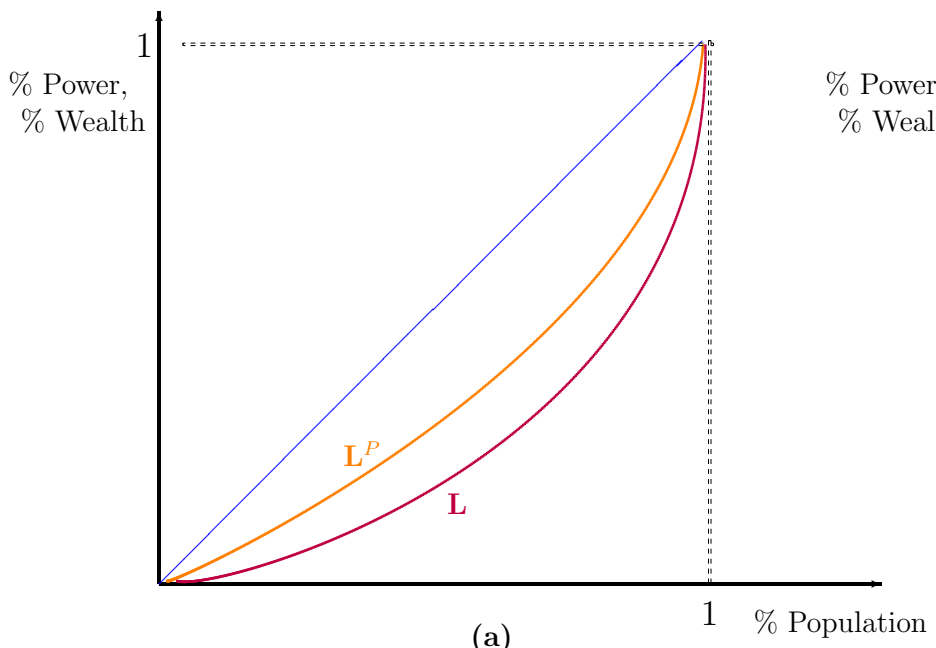

(a)

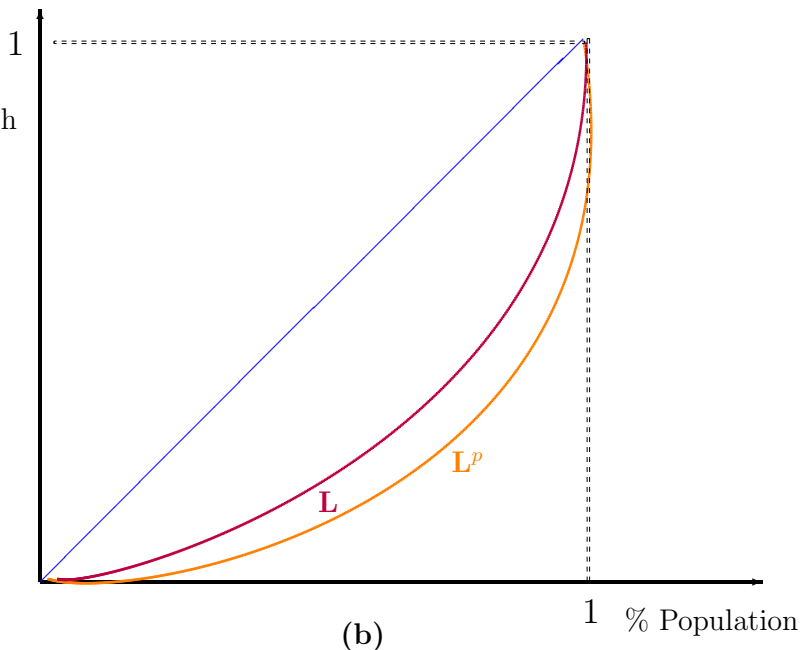

(b)

Figure 1: Political Lorenz Curves with Elitist Bias. (a) exhibits dampened bias. (b) exhibits pronounced bias.

and Assumptions (A1) and (A2). Consequently, standard results (e.g., Rothstein (1990), Gans and Smart (1996)) show that the Median Voter Theorem applies for these preferences. Hence, each period, the rationalized policy is found to be the most preferred policy of the (weighted) median type $i=\mu\left(\omega_{t}, \alpha\right)$ defined by the midpoint of the Political Lorenz curve:

$$
L^{p}(\mu(\omega, \alpha) ; \alpha, \omega)=\frac{\int_{0}^{\mu(\omega, \alpha)} y(i, \omega)^{\alpha} d i}{\int_{0}^{1} y(i, \omega)^{\alpha} d i}=\frac{1}{2}
$$

In the subsequent sections, it will sometimes prove more convenient to consider inference over $\mu(\omega, \alpha)$ directly rather than over $\alpha$. The determination of $\mu(\omega, \alpha)$ is shown in Figure 3 for a particular $\alpha>0$. The resulting policy would be:

$$
a_{t}=1-\left(\bar{y}\left(\omega_{t}\right)\right)^{\frac{1-\rho}{\rho}} /\left(y\left(\mu(\omega, \alpha), \omega_{t}\right)\right)^{\frac{1}{\rho}} .
$$

This policy is increasing in the identity of the median voter. Moreover, one can check that the median voter's type $\mu(\omega, \alpha)$ is increasing in the bias $\alpha$, and so the observer could back out $\alpha$ precisely from observed policy. Of course this presumes the observer knows the exact preference profile which he does not. He must therefore back out the set of biases consistent with his knowledge limited to knowing only that the preference profile is admissible (satisfies (A1) and (A2)). This is our focus of the rest of the way.

\subsection{A Benchmark Result}

The canonical vote share rule in (1) is a special case of a vote share rule satisfying 


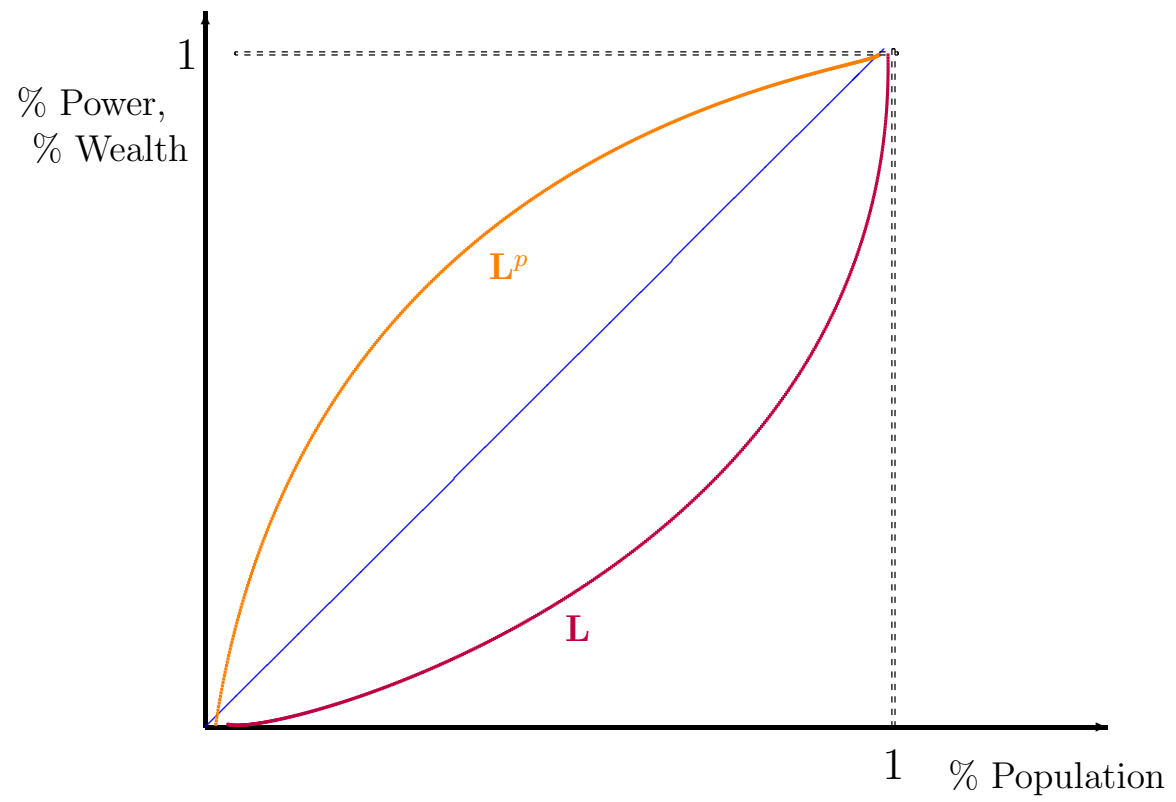

Figure 2: Political Lorenz Curve with Populist Bias

(B1) (Income Monotonicity). Given $\lambda$, a state $\omega$, and a real value $\tilde{y}$ of the function $y$, the density function

$$
\lambda(\tilde{y} ; \alpha) / \int_{y(0, \omega)}^{y(1, \omega)} \lambda(x ; \alpha) d F(x ; \omega)
$$

is assumed to be increasing in income level $\tilde{y}$ if $\alpha>0$, decreasing in income if $\alpha<0$; constant across income levels if $\alpha=0$.

(B2) (Strict Single Crossing) The density function given in (B1) satisfies strict single crossing in $(\alpha ; \tilde{y})$.

In the sequel, any references to a vote share rule will be assumed to satisfy (B1) and (B2). Axiom (B1) asserts that the political power of a citizen varies with his income $\tilde{y}$, and the direction taken by $\lambda$ depends on the sign of $\alpha$. Political power is increasing in income if $\alpha>0$, decreasing if $\alpha<0$, and invariant to income if $\alpha=0$. (B2) guarantees monotonicity of the Political Lorenz Curve in $\alpha$ as asserted below.

Lemma 1 For any Political Lorenz Curve $L^{P}$ corresponding to a vote share rule $\lambda$ satisfying (B1) and (B2), the following holds: for every $j \in(0,1)$ and each $\omega$,

$$
L^{P}\left(j ; \alpha_{2}, \omega\right)<L^{P}\left(j ; \alpha_{1}, \omega\right) \quad \forall \alpha_{1}<\alpha_{2} .
$$




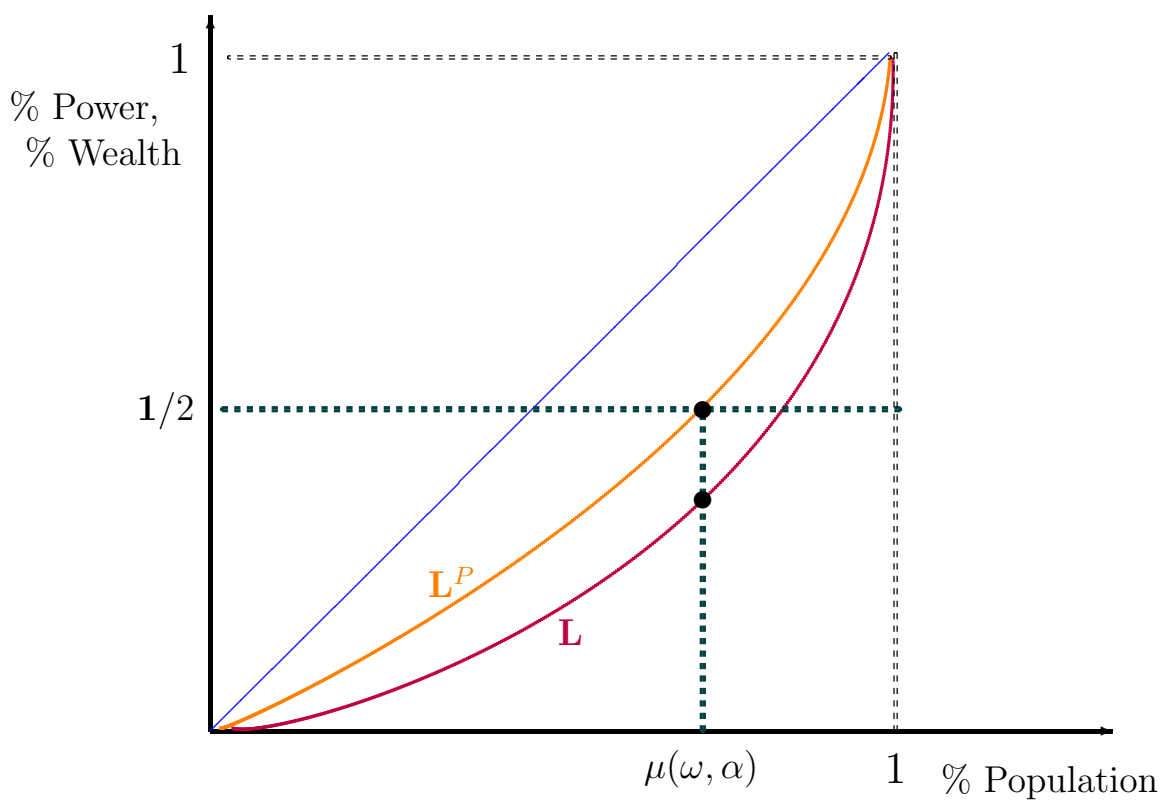

Figure 3: Identifying a Pivotal Voter under an Elitist Bias

The proof is in the Appendix. Under the Lemma, the absolute value $|\alpha|$ can be used to measure the intensity of the bias. Larger positive values correspond to greater elitism in the bias greater political inequality with weight accorded to wealth. A more negative $\alpha$ corresponds to greater populism - again greater political inequality but in reverse.

Definition 1 For any vote share rule $\lambda$, a policy $a$ is said to be a $\alpha$-Weighted Majority Winner $(W M W)$ in state $\omega$ under admissible profile $U$ if, for all policies $\hat{a}$,

$$
\int_{i \in\{j: U(j, \omega, a) \geq U(j, \omega, \hat{a})\}} \lambda(y(i, \omega) ; \alpha) d i \geq 1 / 2 \int_{0}^{1} \lambda(y(i, \omega) ; \alpha) d i
$$

In other words, an $\alpha$-weighted majority winner, or $\alpha$-WMW, is a policy that survives against all others in a majority vote when each type $i$ is allocated $\lambda(y(i, \omega) ; \alpha)$ votes and the preference profile is given by $U$.

If, in fact, the preference profile $U$ were known precisely to the outside observer, then $\alpha$ could be inferred precisely from observed policies that are generated from $\alpha$ (via the weighting function $\lambda$ ). But because $U$ is not known, it is natural to ask whether observed policies might be "rationalized" by a wealth bias $\alpha$ under some admissible preference profile $U$. 
Definition 2 Given vote share rule, a wealth bias $\alpha$ rationalizes the observer's data $\left\{a_{t}, \omega_{t}\right\}_{t=1}^{T}$ if there exists an admissible preference profile $U$ such that for each $t=1, \ldots, T, a_{t}$ is an $\alpha$ weighted majority winner in state $\omega_{t}$ under $U$.

Recall that admissible preferences are those that satisfy (A1) and (A2). One can now ask whether a given $\alpha$ could rationalize an observed collection of policies. This is addressed by the following preliminary result that serves as a benchmark for rest of the paper.

Universal Bias Principle. Let $\lambda$ be a vote share rule and $\left\{a_{t}, \omega_{t}\right\}_{t=1}^{T}$ be any observable data. Let $\alpha$ be any given wealth bias. Then $\alpha$ rationalizes $\left\{a_{t}, \omega_{t}\right\}_{t=1}^{T}$.

Without further information about preference orderings, the Universal Bias Principle (UBP) tells us that all wealth biases can be generated by the model. In particular, one can say nothing specific about political inequality, whether it exists or whether its magnitude is large. Since, among all other $\alpha$, the unbiased polity with $\alpha=0$ can also rationalize policy data, it cannot be ruled out. The UBP also reveals that, clearly, single crossing is not necessarily too restrictive since relaxing it will not help.

The proof of UBP is fairly simple, yet to our knowledge the result has not been demonstrated before. Basically, standard results (e.g., Rothstein (1990), Gans and Smart (1996)) show that for any admissible profile $U$, a Median Voter Theorem applies. Namely, any bias weight $\alpha$ admits a weighted majority-winning policy in each state $\omega$. This policy is found to be the most preferred policy of the median type $i=\mu(\omega, \alpha)$ in the Political Lorenz distribution (recall Equation (4)). All that remains is to find an explicit, admissible payoff function $U$ such that the preferred policy of type $i=\mu(\omega, \alpha)$ in state $\omega$ is precisely the one that is observed in

the data. There are many such $U_{\mathrm{s}}$, for instance, $U(i, \omega, a)=-\frac{1}{2}[a-(i-\mu(\omega, \alpha)+\Psi(\omega))]^{2}$, where $\Psi$ is any function such that $\Psi\left(\omega_{t}\right)=a_{t}$ for each observation date $t$. It is easy to verify that $U$ is single-peaked in $a$, continuous and strictly single-crossing in $(a ; i)$, with the preferred policy for $\mu\left(\omega_{t}, \alpha\right)$ as $a_{t}$ for each $t$.

\section{Rationalizing Policy and Polling Data}

In light of the Universal Bias Principle, there are two possible ways to add predictive content. First, one could add observations on preferences directly from, say, polling data. Second, one can pare down the class of admissible profiles. Here we consider the first option.

Poll data constitute a natural source of information. This section shows how policy and polling outcomes together can be used to derive a useful system of cross restrictions that reveal information about political bias. For simplicity, we consider a simple tracking poll that compares the chosen policy $a_{t}$ at each $t$ to a fixed collection of ordered alternatives 
$a^{1}<a^{2}<\ldots<a^{N}$. Typically, these will be some much discussed policy alternatives, always on the table but not necessarily adopted. Obviously there are a number of ways the comparison can be done. We consider a number of different polling formats and show that each fundamentally lead to the same inferences on the bias. ${ }^{8}$

The subsequent analysis makes use the following notation. First, define a fictitious policy choices $a^{N+1}=\max a+\epsilon$ and $a^{0}=\min a-\epsilon$ for some $\epsilon>0$. Next, define:

$$
n_{t}^{*}=\min \left\{n=1, \ldots, N+1: a^{n}>a_{t}\right\} .
$$

By this definition, $a^{n_{t}^{*}}$ is the closest "right-wing" alternative to the observed policy $a_{t}$ (i.e., closest policy to the right of $a_{t}$ ), while $a_{t}^{n_{t}^{*}-1}$ is the closest "left-wing" alternative to the observed policy $a_{t} \cdot{ }^{9}$ The fictitious policy $a^{N+1}$ and $a^{0}$ ensure that both $n_{t}^{*}$ and $n_{t}^{*}-1$ are well defined. When there is a single alternative to the equilibrium then the poll data conforms to a simple binary tracking poll such as the one by Rasmussen tracking support for repeal of the so-called "Obamacare" landmark healthcare legislation. ${ }^{10}$ A sample is given below.

\begin{tabular}{|c|c|c|}
\hline$\underline{\text { Date }}$ & Favor Repeal & Oppose Repeal \\
\hline Mar 31-Apr 1, 2012 & $54 \%$ & $40 \%$ \\
\hline Mar $17-18$ & $56 \%$ & $39 \%$ \\
\hline Mar 3-4 & $53 \%$ & $42 \%$ \\
\hline Feb $18-19$ & $53 \%$ & $38 \%$ \\
\hline $\mathrm{Feb} 4-5$ & $54 \%$ & $41 \%$ \\
\hline$\vdots$ & $\vdots$ & \\
\hline Mar 23-24, 2010 & $55 \%$ & $42 \%$ \\
\hline
\end{tabular}

In the table, $a_{t}$ is the equilibrium policy of "oppose repeal." ${ }^{11}$ When $N \geq 2$, tracking polls can be formulated in a variety of ways. In the next subsection we discuss some of the most common formats. The main inference results, it turns out, will hold in all such cases.

\footnotetext{
${ }^{8}$ For related literature on polling, see Section 6.

${ }^{9}$ Implicitly, $n_{t}^{*}$ depends on the realized policy $a_{t}$ but we omit the dependence in the notation for simplicity.

${ }^{10}$ Source: "Health Care Law," April 2, 2012 in www.rasmussenreports.com

${ }^{11}$ Recall that the basic inference does not require that the $T$ observation dates coincide with elections.
} 


\subsection{Polling Formats}

Here, three polling formats in which observed policies may be compared with alternatives. To keep the inference deterministic, we assume that there is no measurement error in any of the following.

Pairwise comparisons. Formally, in each period $t$, there are $N$ binary polls taken, each of which pit the observed $a_{t}$ against an alternative policy. The poll data consist of a sequence $\left\{p_{t}^{n}\right\}_{n=1, t=1}^{N, T}$ such that $p_{t}^{n}$ is the support rate at date $t$ that (weakly) favors observed policy $a_{t}$ against alternative $a^{n}$.

Pairwise comparisons are common in electoral polling. For example, in the 2012 primary presidential election in the U.S. many tracking polls pitted President Obama against various GOP challengers. ${ }^{12}$

$\boldsymbol{N}$-ary comparisons. Another common polling format provides preference data pitting the current policy against a variety of options simultaneously. For example, a USA Today/Gallop Poll (Figure 4) pits the status quo that incorporates the 2003 tax cut under President Bush against an expiration option (in which taxes then revert to their pre-2003 levels), as well as a number of more limited expiration options (see USA Today/Gallop Poll (2010)).

\section{COMBINED RESPONSES (Q.13/15): BASED ON NATIONAL ADULTS}

2010 Nov 19-21

\author{
Allow tax cuts to expire for all Americans \\ Set new limits on wealthy Americans' income eligible for \\ lower tax rates \\ (Set limit at $\$ 250,000$ and over) \\ (Set limit at \$500,000 and over) \\ (Set limit at \$1,000,000 and over) \\ (Set limit, unspecified)
}

Keep tax cuts for all regardless of income

No opinion
13

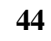

(26)

(12)

(5)

(1)

40

3

Figure 4: USA Today/Gallop Poll Comparing Alternative Tax Policies

Generally, the $N$-ary polling yields a vector $\left(q_{t}^{n}\right)_{n=1, \ldots N}$ of support rates each period where $q_{t}^{n}$ denote the support rate for option $n$ against the other options, including the current policy

\footnotetext{
${ }^{12}$ For Obama versus Romney, see

http://townhall.com/polltracker/statpollsummary/general-election-obama-vs-romney, for Obama versus Santorum, see http://townhall.com/polltracker/statpollsummary/obama-vs-santorum, and for Obama versus Gingrich see http://townhall.com/polltracker/statpollsummary/obama-vs-gingrich.
} 
$a_{t}$. The support rate for $a_{t}$ is given by $1-\sum_{n=1}^{N} q_{t}^{n}$.

Directional polls. Many polls pit the current status quo against a direction of change rather than a specific alternative. To illustrate, consider a sample in Figure 5 from a long time series of tracking poll data conducted by Gallop starting from $1956 .^{13}$

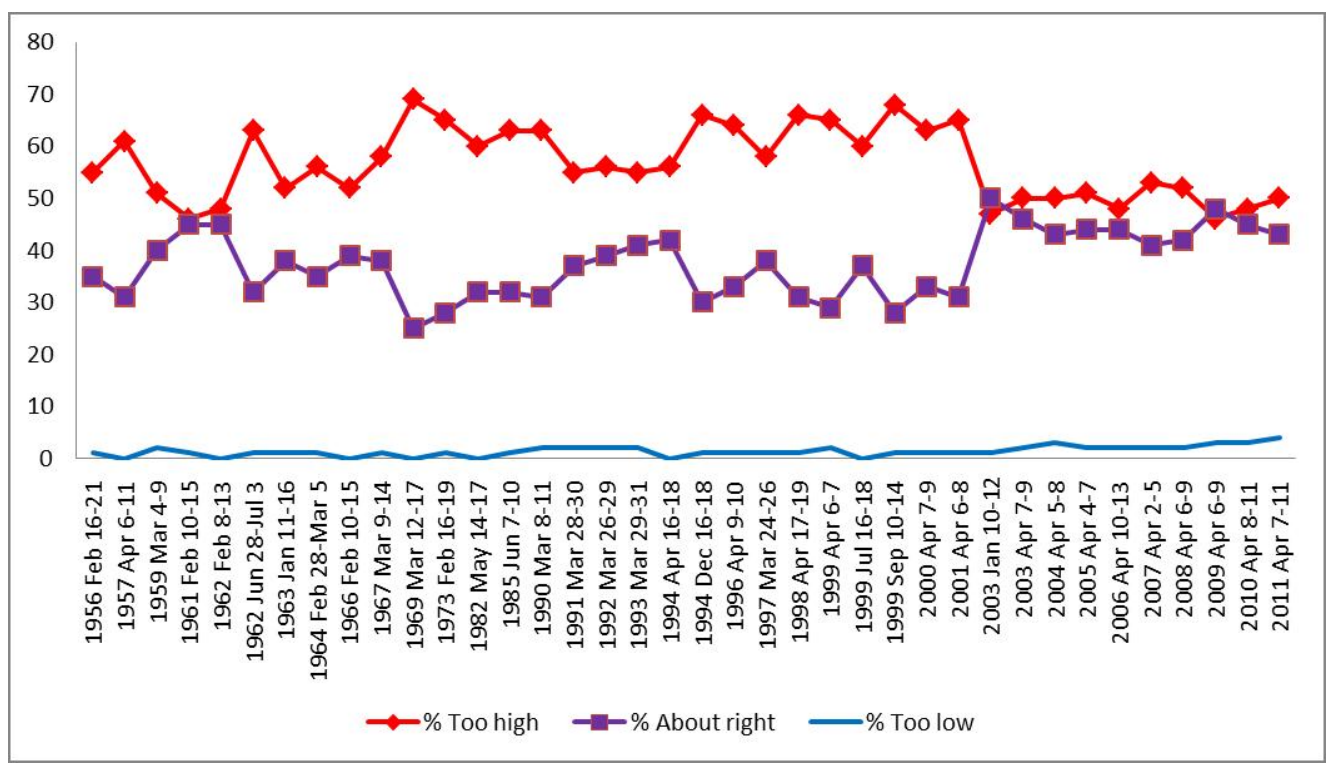

Figure 5: Gallop Tracking Poll on Directional Preferences for Tax Rates

The poll in Figure 5 implicitly compares the status quo tax rates at each date to a given finite collection of tax rates that are to the right or the left of the equilibrium tax rate. For tractability, assume that the alternatives to $a_{t}$ are commonly known to come from the polling set $\left\{a^{1}, \ldots, a^{N}\right\}$. In general one can summarize the directional data in two numbers as follows. Let $r_{t}$ denote the total support rate for all $a \leq a_{t}$ against all policies to the right of $a_{t}$. Let $\ell_{t}$ denote the total support rate for all $a \geq a_{t}$ against all policies to the left of $a_{t}$. For instance, in Figure 5 we can define the tax rate as $(1-a)$ such that a higher $a$ corresponds to a lower tax rate. Then, the support rate for a higher (lower) $a$ - those who think taxes are "too high" ("too low") - is given by $1-r_{t}\left(1-l_{t}\right)$. The support for $a_{t}$ - those who think taxes are "about right" - is then given by $\left(l_{t}+r_{t}-1\right)$.

If all three types of polls were conducted at once and were consistent with one another under our preference assumptions (A1) and (A2), it would turn out that

$$
\begin{aligned}
& r_{t}=p_{t}^{n_{t}^{*}}=1-\sum_{n \geq n_{t}^{*}} q_{t}^{n} \\
& \ell_{t}=p_{t}^{n_{t}^{*}-1}=1-\sum_{n \leq n_{t}^{*}-1} q_{t}^{n}
\end{aligned}
$$

\footnotetext{
${ }^{13}$ See Gallop Poll Social Series (2011).
} 
The first part of each string equates each directional support rate with the binary supports for $a_{t}$ against its closest right-wing and closest left-wing alternatives, respectively. ${ }^{14}$ The second part equates the binary support rates to the sum of all individual support rates to the right of $a_{t}$ in the $N$-ary poll. Notice that when there is only one alternative to the right and to the left of $a_{t}$, then all three polling formats are equally informative. However, when there are more than one alternatives to each side, directional polls are less informative than data on either pairwise or $N$-ary comparisons, both of which are complementary — neither is more informative than the other.

In fact, the main results of this section show that inference requires that only directional data, the least informative of the three, be observed. Hence, any of the three formats will yield the same inference on the bias.

\subsection{Rationalizing both Policy and Polling Data}

The definition of a rationalizing bias can now be extended to polling data for each of the three polling formats described above. The definition below applies to pairwise data; the extension to the other two formats are given in a footnote following the definition.

Definition 3 Given a vote share rule $\lambda$, a wealth bias $\alpha$ rationalizes the policies $\left\{a_{t}, \omega_{t}\right\}_{t=1}^{T}$ and pairwise poll data $\left\{p_{t}^{n}\right\}_{t=1, n=1}^{T, N}$ if there exists an admissible $U$ such that

(i) $\forall t, a_{t}$ is an $\alpha$-Weighted Majority Winner in state $\omega_{t}$ under $U$, and

(ii) $\forall t \forall n$ with $a^{n} \neq a_{t}, U$ satisfies

$$
p_{t}^{n}=\left|\left\{i: U\left(i, \omega_{t}, a_{t}\right) \geq U\left(i, \omega_{t}, a^{n}\right)\right\}\right|
$$

This definition extends the earlier notion of rationalizing weights to one that includes polling data. Part (i) is the policy-consistency requirement as before. Part (ii) is a pollconsistency requirement. It requires that the underlying preference profile $U$ be admissible and consistent with both types of data. With minor modifications, the definition can also be applied to $N$-ary comparisons and directional polling. ${ }^{15}$

\footnotetext{
${ }^{14}$ Recall, $n_{t}^{*}$ is the closest right-wing and $n_{t}^{*}-1$ the closest left-wing alternatives, respectively, and if either $n_{t}^{*}$ or $n_{t}^{*}-1$ is a fictitious alternative $N+1$ or 0 , set $p^{0}=1, p^{N+1}=1$, resp., and $q^{0}=0, q^{N+1}=0$, resp.

${ }^{15}$ For $N$-ary comparisons, replace (ii) in the Definition with: $\forall t \forall n, U$ satisfies

$$
q_{t}^{n}=\left|\left\{i: U\left(i, \omega_{t}, a^{n}\right) \geq U\left(i, \omega_{t}, a\right) \quad \forall a \in\left\{a_{t}, a^{1}, \ldots, a^{N}\right\}, a \neq a^{n}\right\}\right| .
$$
}

For directional polls, replace (ii) with: $\forall t \forall n, U$ satisfies

$$
r_{t}=\left|\left\{i: U\left(i, \omega_{t}, a_{t}\right) \geq U\left(i, \omega_{t}, a\right) \quad \forall a \in\left\{a^{1}, \ldots, a^{N}\right\}, a>a_{t}\right\}\right| .
$$




\subsection{A Characterization}

Recall from (4) that $\mu(\omega, \alpha)=j$ is the pivotal voter in state $\omega$ under the bias $\alpha$. We now state our first main result using pairwise data.

Theorem 1 Let $\lambda$ be a vote share rule and let $\left\{a_{t}, \omega_{t}\right\}_{t=1}^{T}$ and $\left\{p_{t}^{n}\right\}_{t=1, n=1}^{T, N}$ be any policy and binary polling data such that $0<p_{t}^{n}<1$ for all $n$ and $t$. Then, any given $\alpha$ rationalizes the policy and poll data if and only if

$$
1-p_{t}^{1}<\ldots<1-p_{t}^{n_{t}^{*}-2}<1-p_{t}^{n_{t}^{*}-1}<\mu\left(\omega_{t}, \alpha\right)<p_{t}^{n_{t}^{*}}<p_{t}^{n_{t}^{*}+1}<\ldots<p_{t}^{N}
$$

The inequalities in (7) include both polling data restriction, testing directly the validity of the model, and a restriction on the bias itself. The latter speaks more directly to the topic of the paper.

The "sufficiency" arguments entail a specific construction of a profile $U$ under which a bias can rationalize the data. The formal arguments appear in the Appendix. The necessary conditions are more intuitive. The inequalities between the support rates in (7) follow directly from admissibility of preferences, i.e., (A1) and (A2). If, for instance, we had $p_{t}^{n_{t}^{*}+1} \leq p_{t}^{n_{t}^{*}}$, then by the strict single crossing property we could find an individual $i \in\left[p_{t}^{n_{t}^{*}+1}, p_{t}^{n_{t}^{*}}\right]$ who weakly preferred $a_{t}^{n_{t}^{*}+1}$ to $a_{t}$ and at the same time weakly preferred $a_{t}$ to $a_{t}^{n_{t}^{*}}$. Since the actions are ordered $a_{t}<a_{t}^{n_{t}^{*}}<a_{t}^{n_{t}^{*}+1}$, this individual's $U$ would violate single peakedness.

The inequalities in $(7)$ bounding $\mu\left(\omega_{t}, \alpha\right)$ can be understood from basic equilibrium logic. Consider the upper bound. Suppose, contrary to (7), that $p_{t}^{n_{t}^{*}}<\mu\left(\omega_{t}, \alpha\right)<1$ holds. Then the fraction $\left(p_{t}^{n_{t}^{*}}, 1\right]$ who prefer the closest right-wing alternative $a_{t}^{n_{t}^{*}}$ would exceed half the weighted vote share. This would place the supporters of $a_{t}^{n_{t}^{*}}$ in a position to have vetoed $a_{t}$, in which case $a_{t}$ could not have been a Weighted Majority Winner. If, in fact, $\mu\left(\omega_{t}, \alpha\right)=p_{t}^{n_{t}^{*}}<1$, then by continuity of $U$ in $i$ (Assumption (A1)), this pivotal voter would be indifferent between $a_{t}$ and $a_{t}^{n_{t}^{*}}$, thus violating single peakedness. Consequently, we must have $\mu\left(\omega_{t}, \alpha\right)<p_{t}^{n_{t}^{*}}$. Similar arguments apply to the lower bound of $\mu\left(\omega_{t}, \alpha\right)$.

This same type of logic applies to other polling formats as well.

Theorem 2 Let $\lambda$ be a vote share rule and let $\left\{a_{t}, \omega_{t}\right\}_{t=1}^{T}$ and $\left\{q_{t}^{n}\right\}_{t=1, n=1}^{T, N}$ be any policy and $N$-ary polling data such that $q_{t}^{n} \in(0,1)$ for all $n$ and $t$. Then any given $\alpha$ rationalizes the

and

$$
\ell_{t}=\left|\left\{i: U\left(i, \omega_{t}, a_{t}\right) \geq U\left(i, \omega_{t}, a\right) \quad \forall a \in\left\{a^{1}, \ldots, a^{N}\right\}, a<a_{t}\right\}\right| .
$$


policy and polling data if and only if

$$
\sum_{n=1}^{n_{t}^{*}-1} q_{t}^{n}<\mu\left(\omega_{t}, \alpha\right)<1-\sum_{n=n_{t}^{*}}^{N} q_{t}^{n} .
$$

Notice that there are no data restrictions, i.e., no analogue of the inequalities in (7). Because each alternative is compared to the rest of the field, single crossing places no restriction on support rates. Given the consistency condition in (6), the following result for directional data is immediate.

Theorem 3 Let $\lambda$ be a vote share rule and let $\left\{a_{t}, \omega_{t}\right\}_{t=1}^{T}$ and $\left\{\left(\ell_{t}, r_{t}\right)\right\}_{t=1}^{T}$ be any policy and directional polling data. Then any given $\alpha$ rationalizes the policy and polling data if and only if

$$
1-\ell_{t}<\mu\left(\omega_{t}, \alpha\right)<r_{t}
$$

\subsection{Bias Bands and Income Inequality}

Given the consistency condition (6) across polling formats, it is enough to look only at the bounding inequality (9) for inference properties. The inequality (9) can, in fact, be translated into bounds on the bias itself. Notice that, since the Political Lorenz Curve $L^{P}$ is decreasing in the weight $\alpha$ (holding $\omega$ fixed), the pivotal function $\mu$ is invertible in the value $\alpha$. Hence, let $M(j, \omega)$ denote the inverse pivotal function, defined as the map that associates pivotal voter $j$ with the wealth bias that would, in fact, yield $j$ as the pivotal voter. ${ }^{16}$ Applying $M$ to the inequalities in (9) yields

$$
M\left(1-\ell_{t}, \omega_{t}\right)<\alpha<M\left(r_{t}, \omega_{t}\right), \quad t=1, \ldots, T .
$$

This defines a bias band, i.e., an upper and lower bound on the bias as indicated in Figure 6. The bounds of the band in the figure are displayed on the vertical axis. In this particular graph, the range of bias band includes 0 , the unbiased weight. It also includes a subinterval of elitist biases, as well as a subinterval of populist ones.

Theorem 1 together with the definition of $M$ implies straightaway:

Corollary 1 Given a vote share rule $\lambda$, a bias $\alpha$ rationalizes the data (under any of the three polling formats) only if

$$
\max _{t=1, \ldots, T} M\left(1-\ell_{t}, \omega_{t}\right)<\alpha<\min _{t=1, \ldots, T} M\left(r_{t}, \omega_{t}\right)
$$

\footnotetext{
${ }^{16}$ Because the set $[\mu, \bar{\mu}]$ of pivotal voters may not include all of $[0,1]$, we extend the domain of $M$ as follows. If $j<\underline{\mu}$, let $M(j, \omega) \equiv M(\underline{\mu}, \omega)$, and if $j>\bar{\mu}$, let $M(j, \omega)=M(\bar{\mu}, \omega)$.
} 


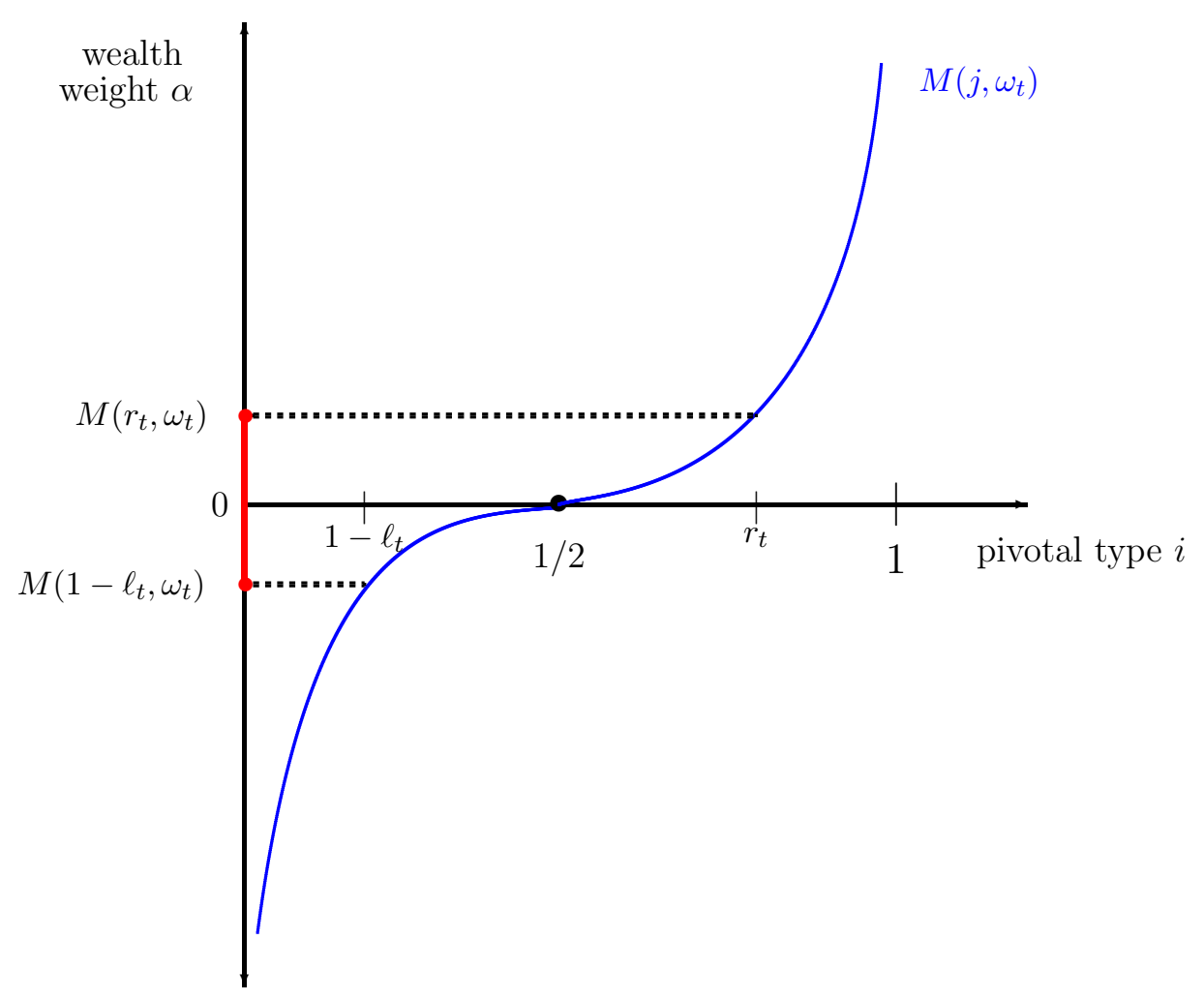

Figure 6: Bias Band and Bounding Function

In other words, observations have a cumulative effect; each observation date serves as a cross check against the observations at other dates. As a result, the effective bias band must shrink - at least weakly.

Clearly, if $\max _{t=1, \ldots, T} M\left(1-\ell_{t}, \omega_{t}\right) \geq \min _{t=1, \ldots, T} M\left(r_{t}, \omega_{t}\right)$ then there is no rationalizing bias. In that case, one or more of the model's assumptions are violated, including the possibility that $\alpha$ is not constant across states $\omega_{t}$.

Moreover, since $M(j, \omega)>0$ iff $j>1 / 2$, it immediately follows that if $\alpha$ rationalizes the data, then $\min _{t} \ell_{t}<1 / 2$ implies an elitist bias: $\alpha>0$. Likewise, if $\alpha$ rationalizes the data, then $\min _{t} r_{t}<1 / 2$ implies a populist bias: $\alpha<0$. Hence, the basic character of the bias can be identified whenever a policy results with only minority support from the population. This is a suggestive finding given that minority policies are often observed.

Notice that the cumulative effect of the data as shown in Corollary 1 allows the observer to make real time refinements to the observer's inference of the lower and upper bounds at each observation date $t$. These are given by $\underline{M}_{t}=\max _{q=1, \ldots, t} M\left(1-\ell_{q}, \omega_{q}\right)$ and $\bar{M}_{t}=$ $\min _{q=1, \ldots, t} M\left(r_{q}, \omega_{q}\right)$, respectively. Using Corollary 1, the observer at date $t$ infers that a bias $\alpha$ rationalizes the data only if $\underline{M}_{t}<\alpha<\bar{M}_{t}$. By construction, these bounds tighten monotonically so that $\underline{M}_{t} \leq \underline{M}_{t+1} \ldots$ and $\bar{M}_{t} \geq \bar{M}_{t+1} \ldots$ 
Furthermore, these "real time" refinements also allow the observer to infer something about the sequence of future pivotal voters. Using (9), the observer at date $t$ forecasts a sequence of upper bounds on the weighted pivotal voter given by

$$
\mu\left(\omega_{t}, \bar{M}_{t}\right), \mu\left(\omega_{t+1}, \bar{M}_{t}\right), \ldots, \mu\left(\omega_{T}, \bar{M}_{t}\right)
$$

and from below,

$$
\mu\left(\omega_{t}, \underline{M}_{t}\right), \mu\left(\omega_{t+1}, \underline{M}_{t}\right), \ldots, \mu\left(\omega_{T}, \underline{M}_{t}\right)
$$

and at each date $q=t, \ldots, T$,

$$
\mu\left(\omega_{q}, \underline{M}_{t}\right)<\mu\left(\omega_{q}, \alpha\right)<\mu\left(\omega_{q}, \bar{M}_{t}\right) .
$$

At $t+1$, the observer refines his forecast, and draws an entirely new inference using $\bar{M}_{t+1}$ and $\underline{M}_{t+1}$, and so on.

Because the income distribution is arbitrary at this point, these forecasted sequences are not generally ordered. They can jump around from date to date. However, we can order them for the case where income distribution is well ordered. To illustrate this point, consider a vote share rule $\lambda$ that has the canonical form in (1). Re-indexing dates if necessary, let $\omega_{1}<\omega_{2}<\cdots<\omega_{T}$ and suppose that income process $y$ has monotone log differences (MLD) in the pair $(i, \omega)$. That is, for any pair of states, $\omega$ and $\hat{\omega}$, the difference $\log y(i, \omega)-\log y(i, \hat{\omega})$ is either increasing in $i$, or decreasing in $i$.

MLD implies basically that relative income differences between individuals is either increasing or decreasing in the state. Many common income processes in the literature satisfy this condition. Using this restriction, income inequality increase may be shown to increase political inequality.

Proposition 1 Suppose that the vote share rule $\lambda$ satisfies the canonical form in (1), and suppose $y$ has monotone log differences in the pair $(i, \omega)$. Suppose income inequality is increasing in $t$, i.e., $L\left(j, \omega_{t+1}\right)<L\left(j, \omega_{t}\right)$ for all $t=1, \ldots, T-1$. Then a bias $\alpha>(<) 0$ rationalizes the data (under any of the three polling formats) only if

$$
\mu\left(\omega_{t+1}, \alpha\right)>(<) \mu\left(\omega_{t}, \alpha\right), \quad \forall t .
$$

Note that when bias is elitist $(\alpha>0)$, increases in $\mu(\omega, \alpha)$ correspond to increasing political inequality; whereas when bias is populist $(\alpha<0)$, decreases in $\mu(\omega, \alpha)$ correspond to increasing political inequality (in this case, favoring the poor). Note, in particular, that if the income inequality is increasing over time, then Proposition 1 implies that the forecasted bounds on the pivotal voter at any given time $t$ are monotonic: either $\mu\left(\omega_{q}, \bar{M}_{t}\right)<\mu\left(\omega_{q+1}, \bar{M}_{t}\right)$ or $\mu\left(\omega_{q}, \bar{M}_{t}\right)>\mu\left(\omega_{q+1}, \bar{M}_{t}\right), \forall q=t, \ldots, T-1$ depending on whether the bound $\bar{M}_{t}$ is elitist $(>0)$ or populist $(<0)$. 


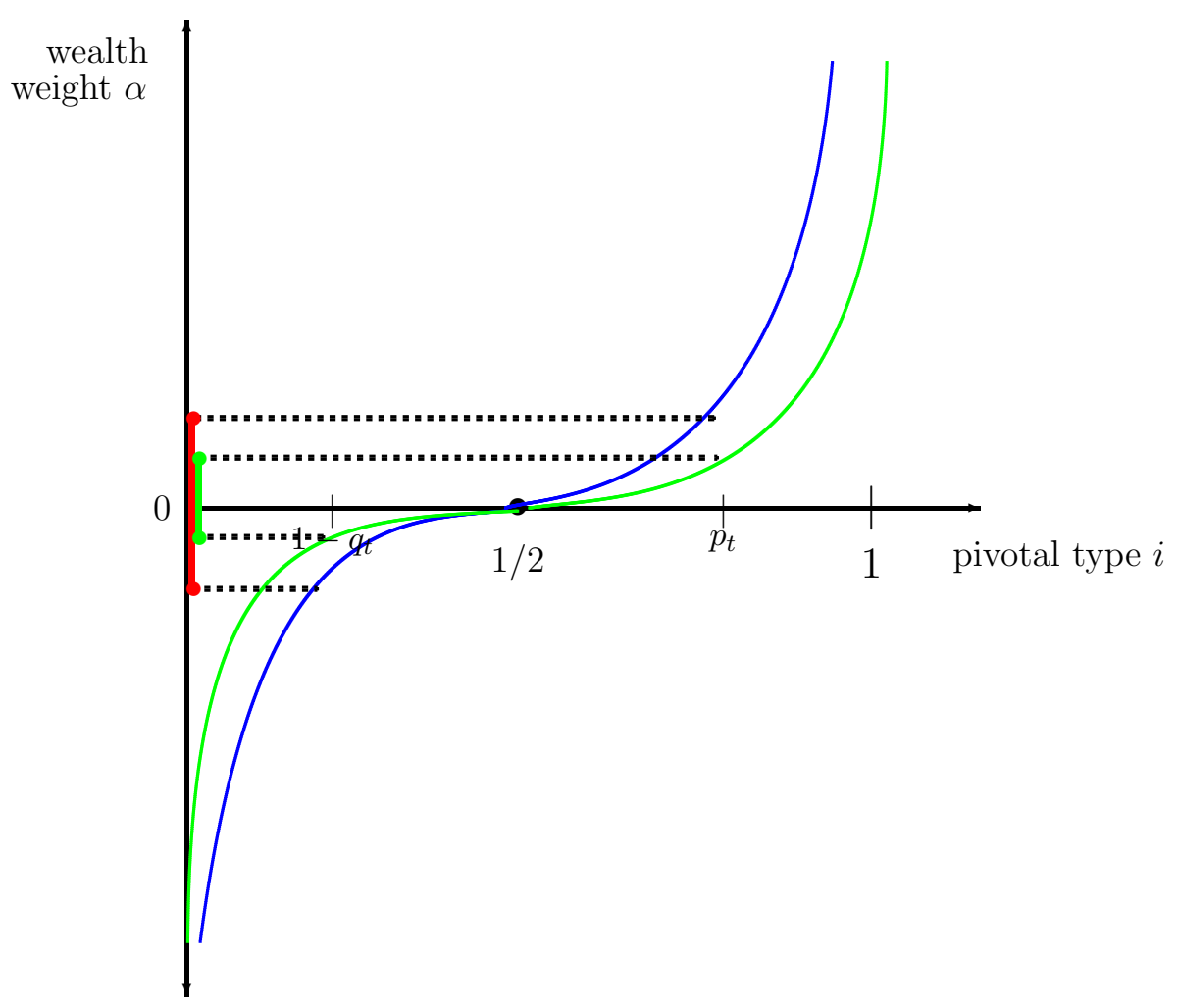

Figure 7: Shrinking Bias Band with Increased Inequality

As a simple comparative statics exercise, consider a ceteris parabis increase in income inequality from $\omega_{1}$ to $\omega_{2}$. Then one can verify that $\left|M\left(j, \omega_{2}\right)\right|<\left|M\left(j, \omega_{1}\right)\right|$ for all $j \neq 1 / 2$. In particular, if 0 (the unbiased weight) belongs to the band, then larger income inequality reduces the size of the band around 0 . Intuitively, this is not surprising if $\alpha>0$ since in that case, the elitist bias can be inferred to have been lower to have off-set the greater income inequality. Somewhat more surprising is the fact that when the band is entirely below 0 (populism), greater inequality moves the band closer to 0 as well. In other words the band becomes less populist implying that wealthier individuals can be inferred to have more political weight than if the distribution were more equal. Why? Because with a populist system, increases in income inequality increase political inequality in favor of the poor. Hence, given $\alpha$, an increase in relative income of the top $10 \%$ translates into an weighted decrease in this group's political power. The wealth bias must then be inferred to be larger to offset this lower political power due to income change. This dual effect of greater income inequality is displayed in Figure 7. 


\section{Restricted Preference Domains}

In this section, we return to the original premise that the observer sees only policy data (i.e., no polling data) and instead, narrow down class of preference profiles. The idea is that observer knows something more, a priori, about the admissible preferences beyond assumptions (A1) and (A2). A narrower class of preference profiles potentially narrows the set of rationalizing biases. To illustrate how additional knowledge of preference could be used by the observer, recall the canonical public goods example in Section 2.4 in which preferences satisfy

$$
U\left(i, \omega_{t}, a_{t}\right)=a_{t} y\left(i, \omega_{t}\right)+\frac{\left[\left(1-a_{t}\right) \bar{y}\left(\omega_{t}\right)\right]^{1-\rho}}{1-\rho} .
$$

Consider two benchmark income processes. (1) the income process satisfies $y\left(i, \omega_{t}\right)=g(i) \omega_{t}$ so that the state $\omega$ summarizes only aggregate growth effects. There are no distributional changes over time. (2) the income process satisfies $\int y(i, \omega) d i=\bar{y}$ so that there are only distributional changes; no aggregate changes in income occur.

Significantly, each of these cases distinctly imply something about $U$. In the first case where only growth effects exist, $U$ satisfies strict single crossing in $(a ; \omega)$. In the second case, where there are only distributional effects, $U$ satisfies separability in income $y(i, \omega)$ and policy $a$ in the sense that $U(i, \omega, a)$ can be expressed as a function $u(y(i, \omega), a)$. These properties have arisen, of course, from a particular payoff function $U$ and a particular income process $y$. However, because these particular functional forms are standard in, for instance, public provision models, we find it useful to examine the inference problem starting from the general properties that generate each. We therefore ask what the observer can infer about bias, starting directly from:

(A3) $U$ satisfies strict single crossing in the pair $(a ; \omega)$ for each $i$.

Assumption (A3) implies that every type's most preferred policy is (weakly) monotone in the state. This monotonicity restriction is fairly common when the policy is a complementary input in the production process. Returning to the canonical public goods model with preferences given by (11), Assumption (A3) can easily be verified in the "growth only" case where $y\left(i, \omega_{t}\right)=g(i) \omega_{t}$. To see what this implies, observe that the most preferred policy of a citizen of type $i$ is

$$
\tilde{\Psi}\left(i, \omega_{t}\right) \equiv 1-\frac{1}{\omega_{t}}\left(\frac{\left(\int g(j) d j\right)^{1-\rho}}{g(i)}\right)^{1 / \rho}
$$

According to the policy definition, the tax rate is $1-\tilde{\Psi}\left(i, \omega_{t}\right)$. The tax rate in the "growth only" case is therefore decreasing in the state. This means that in a growing economy $\left(\omega_{t+1}>\omega_{t}\right)$ with no distributional changes, each voter's ideal tax rate is decreasing over time. This does not imply that the observed tax rates are decreasing since the political power itself can move toward voter-types who prefer relatively higher tax rates. 
Accordingly, the definition of rationalizability must now be modified in the obvious way to include the restriction (A3). Formally,

Definition 4 A given $\alpha$ rationalizes policy data $\left\{a_{t}, \omega_{t}\right\}_{t=1}^{T}$ in the class of admissible preferences satisfying (A3) if there exists an admissible preference $U$ satisfying (A3) such that for each $t=1, \ldots, T, a_{t}$ is a $\alpha$-weighted majority winner under $U$.

Theorem 4 Let $\lambda$ be a vote share rule and let $\left\{a_{t}, \omega_{t}\right\}_{t=1}^{T}$ be any policy data. Then a given $\alpha$ rationalizes policy data in the class of admissible preferences satisfying (A3) if for each pair of observed states $\omega_{t}, \omega_{\tau}$ with $\omega_{t}>\omega_{\tau}$,

$$
a_{t}<a_{\tau} \Longrightarrow \mu\left(\omega_{t}, \alpha\right)<\mu\left(\omega_{\tau}, \alpha\right)
$$

A consequence of Theorem 4 is that any policy data increasing in the observed state can be rationalized by any $\alpha$. However, if the data ever decrease in the state, then certain $\alpha$ may not rationalize the data. For instance,

Corollary 2 Let $\left\{a_{t}, \omega_{t}\right\}$ be any policy data such that the observed policies decrease whenever the state increases. Then the unbiased weight, $\alpha=0$, does not rationalize the data in the class of admissible preferences satisfying (A3).

The sufficiency proof of Theorem 4 requires a constructive argument just as in Theorem 1. An admissible $U$ must be constructed to satisfy the preference axioms while, at the same time, match the policy data on the observed path whenever type $i$ is the pivotal voter, $\mu(\omega, \alpha)$. The construction is complicated in this case by the fact that each citizen's optimal policy rule $\tilde{\Psi}\left(i, \omega_{t}\right)$ must be weakly increasing in the state in order to satisfy (A3), even as the actual policy data might be decreasing in the state. To overcome this, we specify a recursive algorithm that exploits the natural bi-monotonicity of the data in $a_{t}$ and $\mu(\omega, \alpha)$ - as required by the hypothesis in Theorem 4. The formal argument is left to the Appendix.

Finally, consider the property:

(A4) $U$ satisfies separability, i.e., $U(i, \omega, a)=u(y(i, \omega), a)$ for all $a$ and $y(\cdot)$.

Whereas (A3) was motivated by the combination of preferences satisfying (11) and a $y$ process with only growth effects, Assumption (A4) is motivated by the combination of (11) 
and a "distribution only" change in the income process: $\int y\left(j, \omega_{t}\right) d j=\bar{y}$. In the canonical model in (11), this implies that citizen-type $i$ 's most preferred policy is

$$
\tilde{\Psi}\left(i, \omega_{t}\right) \equiv 1-\frac{\bar{y}^{(1-\rho) / \rho}}{\left(y\left(i, \omega_{t}\right)^{1 / \rho}\right.}
$$

In this case, the citizen's most preferred tax rate depends on $i$ and $\omega_{t}$ exclusively through income $y\left(i, \omega_{t}\right)$. In particular, each citizen's preferred tax $\left(1-\tilde{\Psi}\left(i, \omega_{t}\right)\right)$ is decreasing in his income. Assumption (A4) captures the general idea that each citizen's preferred policy rule varies only in his income. Substituting (A4) in place of (A3), we obtain a final result.

Theorem 5 Let $\lambda$ be a vote share rule and let $\left\{a_{t}, \omega_{t}\right\}_{t=1}^{T}$ be any policy data. Then a given $\alpha$ rationalizes policy data in the class of admissible preferences satisfying (A4) if and only if for any pair of observations,

$$
a_{t}<a_{\tau} \Longrightarrow y\left(\mu\left(\omega_{t}, \alpha\right), \omega_{t}\right)<y\left(\mu\left(\omega_{\tau}, \alpha\right), \omega_{\tau}\right)
$$

According to the Theorem, whenever policy is observed to decrease, the weighted median income must decrease as well. To see what this means in the "pure distribution" case, suppose that an increase in inequality over time leads to a drop in median income. In that case a decrease in the observed tax rate would imply a polity with an elitist bias (recall that the tax rate is $1-a_{t}$ in each date). This is consistent with results of Bénabou $(1996,2000)$ that show under an elitist polity, increased inequality is associated with lower levels of redistribution toward the poor.

\section{A Parametric Model of Wealth Bias}

So far, the paper focuses on the reduced-form inference problem, working backward from policies in order to infer bias with minimal modeling structure. This section explores a parametric theory of bias based on a standard model of political competition. The theory is applied to our canonical example in Section 2 and is consistent with the inferential model in the sense that something akin to a vote share can be identified by the model, and, moreover the equilibria are observationally equivalent to policies that are weighted majority winners under this rule.

There are two office-seeking candidates who compete for votes by announcing policies $a^{1}$ and $a^{2}$, resp. The winner implements his announced policy. Ties default to $a^{1}$. Each candidate's success depends on how many of his supporters turn out to vote. Mobilization depends on voters' enthusiasm for a candidate which, in turn, depends on campaign contributions. ${ }^{17}$

\footnotetext{
${ }^{17}$ A related model by Campante (2011) is discussed in the literature review.
} 
After observing the policy platforms, each citizen chooses first how much to contribute to his preferred candidate, then chooses whether to vote. Both voting and contributing are motivated by one's sense of civic duty and enthusiasm for a candidate. This is modeled in a fairly standard way, given the literature. ${ }^{18}$

Denote the payoff to an individual with income $\tilde{y}$ who votes for and contributes $z$ to his preferred candidate by

$$
V(\tilde{y}, \omega, a, z)+\sigma .
$$

Here, civic duty from voting is given by an additively separable term $\sigma \in \mathbb{R}$ (which could be negative), and so he votes if $\sigma \geq 0$. His civic duty from contributing $z$ is captured by function $V$. Conditional on voting/contributing, the individual directs his vote/contribution to candidate $j$ if $V\left(\tilde{y}, \omega, a^{j}, z\right)>V\left(\tilde{y}, \omega, a^{-j}, z\right)$.

We assume that $V$ has a parametric form that generalizes the public good economy of Section 2.3. Formally, assume a payoff given by

$$
\left[c_{i}^{1-\rho}+\beta z_{i}^{1-\rho}\right]^{\frac{1}{1-\rho}}+\frac{1}{1-\rho} G^{1-\rho}, \rho \in[0,1),
$$

subject to $c_{i}+z_{i} \leq a y(i, \omega)$ and $G=(1-a) \bar{y}(\omega)$. Here, $\beta>0$ is a term that weights the effect of a campaign contribution $z_{i}$ and, as before, $c_{i}$ is private consumption, $\bar{y}(\omega)$ is aggregate income, and $G$ a public good funded directly by tax revenue (with tax rate $1-a$ ). Notice that when $z_{i}=0$ we have the parametric payoff in Section 2.3. Using $c_{i}=a y(i, \omega)-z_{i}$ in Equation (15) then gives us the payoff of the form $V(y(i, \omega), \omega, a, z)$ specified in equation (14). By a straightforward calculation, the optimal choice of $z$ to maximize $V$ is

$$
z^{*}\left(y(i, \omega), a^{*}\right)=a^{*} \frac{\beta^{1 / \rho}}{1+\beta^{1 / \rho}} y(i, \omega) .
$$

where $a^{*}$ is the anticipated winning policy between $a^{1}$ and $a^{2}$. It is not difficult to verify that $V\left(\tilde{y}, \omega, a, z^{*}\left(y(i, \omega), a^{*}\right)\right)$ is admissible (satisfies (A1) and (A2)).

Now consider a technology for campaign contributions in which every contribution of $z_{i}$ results in an effective contribution of $z_{i}^{\alpha}$ (due perhaps to costly processing and collection costs). The payoff $V$ remains the same, however, since we assume that $i$ 's feeling of civic duty is based on his actual contribution sacrificed rather than its net effect. In this case, i's effective (as opposed to his original) contribution is

$$
z_{i}^{\alpha}=\left(a^{*} \frac{\beta^{1 / \rho}}{1+\beta^{1 / \rho}} y(i, \omega)\right)^{\alpha} \equiv\left(a^{*}\right)^{\alpha} \lambda(y(i, \omega) ; \alpha)
$$

Notice that this function $\lambda$ is of the same form as the canonical vote share function in Section 2.

\footnotetext{
${ }^{18}$ Using civic duty as a rationale for voting is common in the political economy literature, especially in setups such as ours where no vote or contribution is pivotal in the continuum. See Riker and Ordeshook (1968) for a classic reference. See Feddersen and Sandroni (2006) for an updated version.
} 
Denote the total amount of money actually received by candidate $j$ as $Z^{j}$. Without loss of generality, consider $a^{1} \leq a^{2}$. Because of single crossing, the campaign contributions received by the candidates in the equilibrium continuation following policy platforms $a^{1}<a^{2}$, are:

$$
\begin{aligned}
& Z^{1}(\omega ; \alpha)=\left(a^{*}\right)^{\alpha} \int_{0}^{x} \lambda(y(i, \omega) ; \alpha) d i, \quad \text { and } \\
& Z^{2}(\omega ; \alpha)=\left(a^{*}\right)^{\alpha} \int_{x}^{1} \lambda(y(i, \omega) ; \alpha) d i
\end{aligned}
$$

where $x$ is the citizen-type who is indifferent between $a^{1}$ and $a^{2}$. If $a^{1}=a^{2}$, then each candidate receives half of total campaign contribution, i.e., $Z^{1}(\omega ; \alpha)=Z^{2}(\omega ; \alpha)$.

As for the voting, one's voting participation depends on the aggregate contributions for the preferred candidate and on the size of the partisan base - the idea being that advertising has a larger effect per capita if it can be concentrated on a smaller group. Assume then that $\sigma$ is a random variable, iid across individuals, with the likelihood of voting given by

$$
\operatorname{Prob}\left(\sigma_{i} \geq 0\right)=\left\{\begin{array}{ll}
\frac{\gamma\left(Z^{1}\right)}{x} & \text { if } i \leq x \\
\frac{\gamma\left(Z^{2}\right)}{1-x} & \text { if } i>x
\end{array} .\right.
$$

Here, $\gamma$ is an increasing function and $x$ and $1-x$ are the fractions of voters targeted by candidate 1 and 2, respectively. Using a Law of Large Numbers logic, the implied vote shares are (resp.) $\gamma\left(Z^{1}\right)$ and $\gamma\left(Z^{2}\right)$. Notice that the marginal effect to the candidate $j$ vote from the marginal income type $x$ is proportional to $\lambda(y(x, \omega) ; \alpha)$. In this sense, $\lambda(y(i, \omega) ; \alpha)$ matters determining $i$ 's relative political power, when considering $i$ 's role at the margin, even though each actual vote is weighted the same for all $i$ supporting the same candidate.

Proposition 2 There exists an equilibrium policy pair $\left(a^{1}, a^{2}\right)$ such that for some citizen-type $i=\mu(\omega, \alpha)$,

$$
a^{1}=a^{2}=a^{*}=\arg \max _{a} U(\mu(\omega, \alpha), \omega, a),
$$

where

$$
U(i, \omega, a)=V\left(y(i, \omega), \omega, a, z^{*}(y(i, \omega), a)\right)
$$

and $\mu(\omega, \alpha)$ satisfies

$$
\int_{0}^{\mu(\omega, \alpha)} \lambda(y(i, \omega) ; \alpha) d i=\int_{\mu(\omega, \alpha)}^{1} \lambda(y(i, \omega) ; \alpha) d i .
$$


In words, it may be shown that each candidate receives the same total contribution, i.e., $Z^{1}=Z^{2}$, hence the same implied vote share, $\gamma\left(Z^{1}\right)=\gamma\left(Z^{2}\right)$, and the equilibrium policy positions converge to the ideal policy of a pivotal voter $x=\mu(\omega, \alpha)$ whose payoff is defined by $U$ above. The arguments are standard, though for completeness we include a proof in the Appendix.

The model therefore produces outcomes that are observationally equivalent to the "detailfree" voting model with weighted vote shares of $\lambda(y(i, \omega) ; \alpha)$. While the channel in this case is campaign contribution, there are alternatives that work through voting participation more directly or through valence (ideological predisposition) of the citizenry. What matters in all such cases is not whether one's vote is explicitly weighted per se, but rather whether a citizen's participation at some level or another is, at the margin, differentiated by his income.

\section{Concluding Remarks: Relating to the Literature}

This paper adapts ideas from revealed preference theory to understand political bias. To assess the bias, we formulate a theory of inference based on an outside observer's direct view of policy rather than on indirect measures such as political participation. The theory associates political bias with the weights on a system of wealth-weighted majority voting.

The idea that political bias can be associated with weights in an implicit voting system has been used elsewhere, albeit in different contexts. For instance, the weights given to valence characteristics in probabilistic voting models (Lindbeck and Weibull (1993)) are commonly associated with bias. Bénabou $(1996,2000)$ explicitly associates bias with wealth-weighted voting in his influential study of the effect of income inequality on incentives for redistribution. We adopt Bénabou's terminology, using "elitist" and "populist" to describe pro-weath and anti-wealth biases, resp.

A more common approach in the literature is one that attributes bias to a specific cause. For example, one prominent theory links bias to differential participation rates among the rich and poor (e.g., Bourguignon and Verdier (2000)). The poor vote less frequently, and so one can argue that wealthier voters have a disproportionate influence on policy.

A second type of theory concerns the effect of campaign contributions, for instance, AustenSmith (1987), Baron (1994), Grossman and Helpman (1996), Prat (2002), Coate (2004), Campante (2011). In these models, the money either "buys" influence directly or it affects policy indirectly by changing the electoral odds toward candidates ideologically predisposed toward the rich. Because contributions skew toward the wealthy, policies are biased in their favor. The contributions model laid out in Section 5 is similar to Campante's. Our setup differs from his in that: (a) a different mobilization technology is used here to generate wealthweighted voting; (b) the present model generates policy convergence, while Campante's model features policy divergence. 
Finally, a third type of theory centers on disenfranchising investments, e.g., Acemoglu and Robinson (2008), made by a wealthy elite in order to disinherit the poor from the political process.

By also looking at polling data, our paper complements a strand in the political science literature on public opinion and political representation (see Manza and Cook (2002) for a comprehensive survey). This literature empirically investigates the linkage between the policy choices and public support as measured in opinion polls. For salient policy issues, the findings typically find positive correlations between policy changes and public support but is generally too coarse in its approach to rule out (or in) particular forms of bias.

Recently, a small and growing literature uses more refined polling data and techniques to ascertain bias. Jacob and Page (2005), for instance, examine tracking polls of the public support by business leaders and by the general public for U.S. foreign policy. They find that policy is most responsive to elite business groups, with little influence from the general public. Gilens (2005) uses a large sample of binary opinion polls between 1981 and 2002 to analyze the statistical association of the public support rates for existing policy across different income groups with subsequent policy choices, and finds that actual policy outcomes strongly reflect the preferences of the rich group, but bear virtually no relationship to the preferences of the poor or middle-income Americans. These findings are echoed by the aforementioned findings of Bartels (2008).

Finally, by "working back" from policy, the present paper follows in the tradition of Afriat (1967) who examines how an individual utility function can be constructed from finite consumption and price data. ${ }^{19}$ However, our model involves aggregation of choice and uses a political system to do so. More typically RPT approaches to aggregation follow the general equilibrium tradition, the classic example being the Sonnenschein-Mantel-Debreu result that checks whether an aggregate excess demand function is consistent with the economy-wide aggregation of optimizing choices. ${ }^{20}$

The RPT paradigm has been applied elsewhere to political choices. Kalandrakis (2010) for instance finds necessary and sufficient conditions for the results of a series of roll call votes to be rationalized by a voter with quasi-concave utility. Degan and Merlo (2009) use micro-level voting data to examine whether the outcomes of simultaneous, multi-candidate elections can be rationalized by ideological voting behavior.

As in the standard RPT mode, one can interpret the observations here as having no intertemporal connection. However, one can also view the observations as coming from a fully dynamic economy populated by infinitely lived citizens. Under the latter interpretation, the data consist of a time series produced by the same underlying polity. Viewed in this way, the present paper extends Boldrin and Montrucchio's (1986) dynamic model of rationalizability

\footnotetext{
${ }^{19}$ See also Varian (1982), Chiappori and Rochet (1987).

${ }^{20}$ References for this result are Sonnenschein (1973), Mantel (1974), Debreu (1974). See also references and recent results in Brown and Kubler (2008) for applications of RPT to general equilibrium theory.
} 
of policy rules by a single agent to the case of political aggregation.

\section{Appendix}

\section{Appendix A: The Dynamic Economy Interpretation}

The presentation in the main text does not specify an explicit intertemporal connection between observations. Extending the analysis to a dynamic economy with infinite-lived forwardlooking decision makers requires adding three ingredients.

1. The states and policy are connected through a transition function $\omega_{t+1}=Q\left(\omega_{t}, a_{t}\right)$, which must be known to the participants and may be partly inferred from the data path $\left\{a_{t}, \omega_{t}\right\}_{t=1}^{T}$ by the outside observer.

2. Forward-looking individuals correctly forecast future economic policies both on and off the equilibrium path. We restrict attention to admissible Markov policy rules, i.e. given any policy data $\left\{a_{t}, \omega_{t}\right\}_{t=1}^{T}$ there exists a function $\Psi: \Omega \rightarrow A$ satisfying

$$
\Psi\left(\omega_{t}\right)=a_{t}, \forall t=1, \ldots, T
$$

The Markov restriction allows for a tractable characterization of the data even as it entails some loss of generality. It seems appropriate in large and anonymous societies where history-dependent enforcement mechanisms would be difficult to implement.

3. The life-time utility is additively separable with a flow payoff $u(\omega, y, a)$ and a known discount factor $\delta \in[0,1)$. In a Markov equilibrium, this implies that for every $(i, \omega, a)$

$$
U(i, \omega, a)=u(\omega, y(i, \omega), a)+\delta U(i, Q(\omega, a), \Psi(Q(\omega, a))) .
$$

A payoff function $U(i, \omega, a)$ satisfying (A1), (A2) and Equation (16) is then referred to as a dynamically admissible preference profile.

Definition 5 A weight $\alpha$ dynamically rationalizes the observer's data $\left\{a_{t}, \omega_{t}\right\}_{t=1}^{T}$ if there exists a dynamically admissible preference profile $U$ and an admissible Markov policy rule $\Psi(\omega)$ such that $\Psi(\omega)$ is an $\alpha$-weighted majority winner in every $\omega$ under $U$.

By definition, dynamic rationalization under a given $U$ implies rationalization under the same $U$. On the other hand, if $\alpha$ rationalizes the observed data under $U$, then it also dynamically rationalizes the data under the flow payoff

$$
u(\omega, y, a)=U(F(\omega, y), \omega, a)-\delta U(F(\omega, y), Q(\omega, a), \Psi(Q(\omega, a)))
$$


and

$$
\Psi(\omega)=\arg \max U(\mu(\omega, \alpha), \omega, a) .
$$

As a result, dynamic-rationalization imposes the same testable restrictions as rationalization as defined in Definition 2 in the main text.

\section{Appendix B: Proofs of the Results}

Proof of Lemma 1 Let $f(i, \alpha, \omega)=\frac{\lambda(y(i, \omega) ; \alpha)}{\int_{0}^{1} \lambda(y(s, \omega) ; \alpha) d s}$. Fix a state $\omega$ and let $\alpha_{2}>\alpha_{1}$. Now define

$$
D(j)=L^{P}\left(j ; \alpha_{2}, \omega\right)-L^{P}\left(j ; \alpha_{1}, \omega\right)=\int_{0}^{j}\left(f\left(i, \alpha_{2}, \omega\right)-f\left(i, \alpha_{1}, \omega\right)\right) d i .
$$

From strict single crossing property, $f\left(i_{1}, \alpha_{2}, \omega\right)-f\left(i_{1}, \alpha_{1}, \omega\right) \geq 0$ implies $f\left(i_{2}, \alpha_{2}, \omega\right)-$ $f\left(i_{2}, \alpha_{1}, \omega\right)>0$ for every $i_{2}>i_{1}$. By definition, $D(0)=0$ and $D(1)=0$. As a result, it cannot be the case that $f\left(i, \alpha_{2}, \omega\right)-f\left(i, \alpha_{1}, \omega\right)>0$ or $f\left(i, \alpha_{2}, \omega\right)-f\left(i, \alpha_{1}, \omega\right)<0$ for almost all $i \in(0,1)$. Consequently, as a function of $i, f\left(i, \alpha_{2}, \omega\right)-f\left(i, \alpha_{1}, \omega\right)$ crosses zero exactly once and from below. This implies that $L^{P}\left(j ; \alpha_{2}, \omega\right)<L^{P}\left(j ; \alpha_{1}, \omega\right)$ for every $j \in(0,1)$.

\section{Proof of Theorem 1.}

As the necessary part was shown in the main text, it remains to show the sufficiency argument.

Sufficiency. Now we suppose that the inequalities in (7) hold and proceed to show that $\alpha$ rationalizes the data. Consider any payoff $U$ of the form

$$
U(i, \omega, a)=-\frac{1}{2}(a-\widetilde{\Psi}(i, \omega))^{2}
$$

where $\widetilde{\Psi}(i, \omega)$ is continuous and increasing in $i$ for every $\omega \in \Omega$. Notice that every $U$ as defined in (17) is admissible. For (A1), observe that $U$ is continuous in $i$ and strictly concave in $a$ (hence single peaked). From the increasing property of $\widetilde{\Psi}$ in $i, U$ is strict single crossing in $(a ; i)$, as required in $(\mathrm{A} 2)$.

We proceed to construct a particular $\widetilde{\Psi}(i, \omega)$ to be consistent with both policy and polling data. For policy data, notice that $\widetilde{\Psi}(i, \omega)$ is the preferred policy choice for type $i$ under $\omega$. By the Median Voter Theorem (for example, Rothstein (1990), Gans and Smart (1996)), $\alpha$ rationalizes the policy data under $U$ in (17) if and only if there exists a $\widetilde{\Psi}$ such that $\widetilde{\Psi}\left(\mu\left(\omega_{t}, \alpha\right), \omega_{t}\right)=a_{t}$.

To prove consistency of $U$ with polling data comparing $a_{t}$ to any alternative $a^{n}$ with $n \geq n_{t}^{*}$, it suffices to assume that the type $p_{t}^{n}$ is indifferent between $a_{t}$ and $a^{n}$, i.e., $U\left(p_{t}^{n}, \omega_{t}, a^{n}\right)=$ 
$U\left(p_{t}^{n}, \omega_{t}, a_{t}\right)$. With some algebra, it reduces to

$$
\widetilde{\Psi}\left(p_{t}^{n}, \omega_{t}\right)=\frac{1}{2}\left(a^{n}+a_{t}\right)
$$

where $\widetilde{\Psi}$ is the function associated with payoff function $U$ as specified in (17). Similarly, when $n \leq n_{t}^{*}-1$, consistency $U$ with polling for $a_{t}$ against $a^{n}$ implies that the type $1-p_{t}^{n}$ is indifferent between $a_{t}$ and $a^{n}$, i.e., $U\left(1-p_{t}^{n}, \omega_{t}, a^{n}\right)=U\left(1-p_{t}^{n}, \omega_{t}, a_{t}\right)$, which reduces to

$$
\widetilde{\Psi}\left(1-p_{t}^{n}, \omega_{t}\right)=\frac{1}{2}\left(a^{n}+a_{t}\right) \text {. }
$$

To prove that $\alpha$ rationalizes the data under admissible payoff function $U$ of the form in (17), it therefore suffices to construct function $\widetilde{\Psi}$ that is continuous and increasing in $i$ and satisfies the equation systems:

$$
\left(\begin{array}{c}
\widetilde{\Psi}\left(1-p_{t}^{1}, \omega_{t}\right) \\
\vdots \\
\widetilde{\Psi}\left(1-p_{t}^{n_{t}^{*}-1}, \omega_{t}\right) \\
\widetilde{\Psi}\left(\mu\left(\omega_{t}, \alpha\right), \omega_{t}\right) \\
\widetilde{\Psi}\left(p_{t}^{n_{t}^{*}}, \omega_{t}\right) \\
\vdots \\
\widetilde{\Psi}\left(p_{t}^{N}, \omega_{t}\right)
\end{array}\right)=\left(\begin{array}{c}
\frac{1}{2}\left(a^{1}+a_{t}\right) \\
\vdots \\
\frac{1}{2}\left(a^{n_{t}^{*}-1}+a_{t}\right) \\
a_{t} \\
\frac{1}{2}\left(a^{n_{t}^{*}}+a_{t}\right) \\
\vdots \\
\frac{1}{2}\left(a^{N}+a_{t}\right)
\end{array}\right) t=1, \ldots, T \text {. }
$$

Hence, fix any on-path $\omega_{t}$. Then $\widetilde{\Psi}\left(i, \omega_{t}\right)$ can be found as a linear spline passing through data points $\left(0, a^{1}\right),\left(1-p_{t}^{1}, \frac{1}{2}\left(a^{1}+a_{t}\right)\right), \ldots,\left(1-p_{t}^{n_{t}^{*}-1}, \frac{1}{2}\left(a^{n_{t}^{*}-1}+a_{t}\right)\right),\left(\mu\left(\omega_{t}, \alpha\right), a_{t}\right),\left(p_{t}^{n_{t}^{*}}, \frac{1}{2}\left(a^{n_{t}^{*}}+\right.\right.$ $\left.\left.a_{t}\right)\right), \ldots,\left(p_{t}^{N}, \frac{1}{2}\left(a^{N}+a_{t}\right)\right),\left(1, a^{N}\right)$. Notice that $\widetilde{\Psi}\left(i, \omega_{t}\right)$ is increasing in $i$ for each $\omega_{t}$.

Since $\widetilde{\Psi}(i, \omega)$ is not restricted off-path, any $\widetilde{\Psi}(i, \omega)$ increasing in $i$ will serve the purpose. For instance, the construction used in the Universal Bias Principle given by $\widetilde{\Psi}(i, \omega)=i-$ $\mu(\omega, \alpha)+\Psi(\omega)$, will work. This concludes the Sufficiency proof.

\section{Proof of Theorem 2.}

Sufficiency. Now we suppose that the inequalities in (8) hold and proceed to show that $\alpha$ rationalizes the data. We use the same construction as in the proof of Theorem 1. Following the discussion there, we only need to construct a modified $\widetilde{\Psi}(i, \omega)$ such that for every $\omega_{t}$ 


$$
\left(\begin{array}{c}
\widetilde{\Psi}\left(q_{t}^{1}, \omega_{t}\right) \\
\widetilde{\Psi}\left(\sum_{n=1}^{2} q_{t}^{n}, \omega_{t}\right) \\
\vdots \\
\widetilde{\Psi}\left(\sum_{n=1}^{n_{t}^{*}-1} q_{t}^{n}, \omega_{t}\right) \\
\widetilde{\Psi}\left(\mu\left(\omega_{t}, \alpha\right), \omega_{t}\right) \\
\widetilde{\Psi}\left(1-\sum_{n=n_{t}^{*}}^{N} q_{t}^{n}, \omega_{t}\right) \\
\vdots \\
\widetilde{\Psi}\left(1-\sum_{n=(N-1)}^{N} q_{t}^{n}, \omega_{t}\right) \\
\widetilde{\Psi}\left(1-q_{t}^{N}, \omega_{t}\right)
\end{array}\right)=\left(\begin{array}{c}
\frac{1}{2}\left(a^{1}+a^{2}\right) \\
\frac{1}{2}\left(a^{2}+a^{3}\right) \\
\vdots \\
\frac{1}{2}\left(a^{n_{t}^{*}-1}+a_{t}\right) \\
a_{t} \\
\frac{1}{2}\left(a_{t}+a^{n_{t}^{*}}\right) \\
\vdots \\
\frac{1}{2}\left(a^{N-2}+a^{N-1}\right) \\
\frac{1}{2}\left(a^{N-1}+a^{N}\right)
\end{array}\right) t=1, \ldots, T .
$$

For any on-path $\omega_{t}, \widetilde{\Psi}\left(i, \omega_{t}\right)$ can be found as a linear spine passing through data points $\left(0, a^{1}\right),\left(q_{t}^{1}, \frac{1}{2}\left(a^{1}+a^{2}\right)\right), \ldots,\left(\sum_{n=1}^{n_{t}^{*}-1} q_{t}^{n}, \frac{1}{2}\left(a^{n_{t}^{*}-1}+a_{t}\right)\right),\left(\mu\left(\omega_{t}, \alpha\right), a_{t}\right),\left(1-\sum_{n=n_{t}^{*}}^{N} q_{t}^{n}, \frac{1}{2}\left(a_{t}+a^{n_{t}^{*}}\right)\right)$, $\ldots,\left(1-q_{t}^{N}, \frac{1}{2}\left(a^{N-1}+a^{N}\right)\right)$. Notice that $\widetilde{\Psi}\left(i, \omega_{t}\right)$ is increasing in $i$ for each $\omega_{t}$. This finishes the proof.

Proof of Proposition 1. Notice that both increased income and political inequality are statement about first-order stochastic orderings. In either case, a distribution under, say, $\omega_{2}$ first-order dominates a distribution under $\omega_{1}$ if the likelihood ratio is increasing. The log-likelihood ratio of $L$ is

$$
\log y\left(i, \omega_{2}\right)-\log y\left(i, \omega_{1}\right)-\log \left(\frac{\int_{0}^{1} y\left(s, \omega_{2}\right) d s}{\int_{0}^{1} y\left(s, \omega_{1}\right) d s}\right),
$$

whereas the $\log$-likelihood ratio of $L^{P}$ is

$$
\alpha\left[\log y\left(i, \omega_{2}\right)-\log y\left(i, \omega_{1}\right)\right]-\log \left(\frac{\int_{0}^{1} y\left(s, \omega_{2}\right)^{\alpha} d s}{\int_{0}^{1} y\left(s, \omega_{1}\right)^{\alpha} d s}\right) .
$$

Now suppose that the log difference of $y$ is increasing in $i$. Then standard argument show that both likelihood ratios are increasing if $\alpha>0$. Similarly, the likelihood ratio for $L$ is increasing, and that for $L^{P}$ is decreasing if $\alpha<0$.

\section{Proof of Theorem 4.}

Sufficiency. Fix any policy data and $\alpha$ that satisfy the implication in (12).

\section{Construction of a Class of Payoff Function}


Now consider a payoff function $U$ of the form

$$
U(i, \omega, a)= \begin{cases}-\frac{1}{2}\left(1+\frac{i}{2}\right)\left(1+\frac{\omega-\min \Omega}{2(\max \Omega-\min \Omega)}\right)(a-\widetilde{\Psi}(i, \omega))^{2} & \text { if } a \leq \widetilde{\Psi}(i, \omega), \\ -\frac{1}{2}\left(1-\frac{i}{2}\right)\left(1-\frac{\omega-\min \Omega}{2(\max \Omega-\min \Omega)}\right)(a-\widetilde{\Psi}(i, \omega))^{2} & \text { if } a \geq \widetilde{\Psi}(i, \omega),\end{cases}
$$

where $\widetilde{\Psi}(i, \omega)$ is continuous and weakly increasing in $(i, \omega)$ for every $i \in[0,1]$ and $\omega \in \Omega$. Notice that $U(i, \omega, a)$ as defined in (20) is continuous in $i$, and is single-peaked in $a$, as required in (A1). Graphically, for each fixed $(i, \omega), U(i, \omega, a)$ in (20) defines an inverse Ushaped curve in $a$, which peaks at $a=\widetilde{\Psi}(i, \omega)$ with a maximum $U(i, \omega, \widetilde{\Psi}(i, \omega))=0$. For fixed $(i, \omega)$, an increase in the value of $\widetilde{\Psi}(i, \omega)$ will lead to a rightward parallel shift of the curve. Alternatively, if we fix the value of the ideal point $\widetilde{\Psi}(i, \omega)$, an increase in $i$ (resp. $\omega$ ) will rotate the curve counterclockwise along the ideal point $\widetilde{\Psi}(i, \omega)$. These properties give a geometric intuition for the fact that $U(i, \omega, a)$ satisfies strict single-crossing in $(a ; i)$ (i.e., (A2)) and in $(a ; \omega)$ (i.e., (A3)). ${ }^{21}$ Formally, we have

Lemma 2 Every $U(i, \omega, a)$ defined in (20) satisfies strict single crossing in $(a ; i)$ and in $(a ; \omega)$.

\section{Proof of Lemma 2:}

We first show that strict single crossing in $(a ; i)$ holds, i.e., if $a_{2}>a_{1}, i_{2}>i_{1}$ and $U\left(i_{1}, \omega, a_{2}\right)-$ $U\left(i_{1}, \omega, a_{1}\right) \geq 0$, then $U\left(i_{2}, \omega, a_{2}\right)-U\left(i_{2}, \omega, a_{1}\right)>0$. Notice that the weak monotonicity of $\widetilde{\Psi}(i, \omega)$ implie that $\widetilde{\Psi}\left(i_{2}, \omega\right) \geq \widetilde{\Psi}\left(i_{1}, \omega\right)$. In addition, $U\left(i_{1}, \omega, a_{2}\right)-U\left(i_{1}, \omega, a_{1}\right) \geq 0$ implies that $\widetilde{\Psi}\left(i_{1}, \omega\right)>a_{1}$, since otherwise the single-peakedness would imply that $U\left(i_{1}, \omega, a_{2}\right)-$ $U\left(i_{1}, \omega, a_{1}\right)<0$. Hence, $\widetilde{\Psi}\left(i_{2}, \omega\right) \geq \widetilde{\Psi}\left(i_{1}, \omega\right)>a_{1}$. We prove the result for two cases. First, $\widetilde{\Psi}\left(i_{2}, \omega\right) \geq a_{2}>a_{1}$. Single-peakedness then implies that $U\left(i_{2}, \omega, a_{2}\right)-U\left(i_{2}, \omega, a_{1}\right)>0$.

Second, $a_{2}>\widetilde{\Psi}\left(i_{2}, \omega\right)>a_{1}$. It then implies that $a_{2}>\widetilde{\Psi}\left(i_{1}, \omega\right)>a_{1}$. From the definition of $U(i, \omega, a)$ in $(20)$, we have

$$
\begin{aligned}
& U\left(i_{2}, \omega, a_{2}\right)=-\frac{1}{2}\left(1-\frac{i_{2}}{2}\right)\left(1+\frac{\omega-\min \Omega}{2(\max \Omega-\min \Omega)}\right)\left(a_{2}-\widetilde{\Psi}\left(i_{2}, \omega\right)\right)^{2}, \\
& U\left(i_{2}, \omega, a_{1}\right)=-\frac{1}{2}\left(1+\frac{i_{2}}{2}\right)\left(1+\frac{\omega-\min \Omega}{2(\max \Omega-\min \Omega)}\right)\left(a_{1}-\widetilde{\Psi}\left(i_{2}, \omega\right)\right)^{2}, \\
& U\left(i_{1}, \omega, a_{2}\right)=-\frac{1}{2}\left(1-\frac{i_{1}}{2}\right)\left(1+\frac{\omega-\min \Omega}{2(\max \Omega-\min \Omega)}\right)\left(a_{2}-\widetilde{\Psi}\left(i_{1}, \omega\right)\right)^{2}, \\
& U\left(i_{1}, \omega, a_{1}\right)=-\frac{1}{2}\left(1+\frac{i_{1}}{2}\right)\left(1+\frac{\omega-\min \Omega}{2(\max \Omega-\min \Omega)}\right)\left(a_{1}-\widetilde{\Psi}\left(i_{1}, \omega\right)\right)^{2} .
\end{aligned}
$$

\footnotetext{
${ }^{21}$ Notice that $U$ as defined in (20) satisfies the strict single crossing property in $(a ; i)$ even if the ideal point $\widetilde{\Psi}(i, \omega)$ is only weakly increasing in $i$. This is in contrast with the construction of the form (17), where a weakly increasing $\widetilde{\Psi}(i, \omega)$ would only imply a (weak) single-crossing property. Because the condition specified in (12) does not rule out a constant path of policy data $\left\{a_{t}\right\}$, the consistency with policy data typically requires a weakly increasing $\widetilde{\Psi}(i, \omega)$. This explains our choice of the form (20) instead of (17).
} 
Since $i_{2}>i_{1}$ and $a_{2}>\widetilde{\Psi}\left(i_{2}, \omega\right) \geq \widetilde{\Psi}\left(i_{1}, \omega\right)>a_{1}$, it follows that $\left(1-\frac{i_{1}}{2}\right)>\left(1-\frac{i_{2}}{2}\right)>0$ and $\left(a_{2}-\widetilde{\Psi}\left(i_{1}, \omega\right)\right)^{2} \geq\left(a_{2}-\widetilde{\Psi}\left(i_{2}, \omega\right)\right)^{2}>0$. As a result, we have $U\left(i_{2}, \omega, a_{2}\right)>U\left(i_{1}, \omega, a_{2}\right)$. Similarly, $\left(1+\frac{i_{2}}{2}\right)>\left(1+\frac{i_{1}}{2}\right)>0$ and $\left(a_{1}-\widetilde{\Psi}\left(i_{2}, \omega\right)\right)^{2} \geq\left(a_{1}-\widetilde{\Psi}\left(i_{1}, \omega\right)\right)^{2}>0$, which implies $U\left(i_{2}, \omega, a_{1}\right)<U\left(i_{1}, \omega, a_{1}\right)$. Combining both, we have

$$
U\left(i_{2}, \omega, a_{2}\right)-U\left(i_{2}, \omega, a_{1}\right)>U\left(i_{1}, \omega, a_{2}\right)-U\left(i_{1}, \omega, a_{1}\right) \geq 0 .
$$

This completes the verification of strict single crossing in $(a ; i)$. As for strict single crossing in $(a ; \omega)$ the proof follows the same steps and so we omit the details.

\section{Construction of a Payoff Function Consistent with Policy Data}

To complete the proof, it suffices to construct $\widetilde{\Psi}(i, \omega)$ such that $\widetilde{\Psi}(i, \omega)$ is continuous, weakly increasing in $(i, \omega)$, and satisfies $\widetilde{\Psi}\left(\mu\left(\omega_{t}, \alpha\right), \omega_{t}\right)=a_{t}$.

We first construct it on the finite observed path. The construction is then extended to the remaining states and types. On the finite path, it is convenient to define monotone indices on $\omega$ and on $i$, respectively. By reordering if necessary, we can define an index $t$ with $t=1,2, \ldots, T$ such that $\omega_{t}<\omega_{t+1}, \forall t<T$. The derived sequence of pivotal decision makers is defined as $\left\{i_{t}\right\}_{t=1}^{T}$ such that $i_{t}=\mu\left(\omega_{t}, \alpha\right)$. For the convenience of extending finite data to the whole range of states and types, we specify two fictional end-point observations as $\left(\omega_{0}, i_{0}, a_{0}\right)=(\min \Omega-1,0, \min A)$ and $\left(\omega_{T+1}, i_{T+1}, a_{T+1}\right)=(\max \Omega+1,1, \max A) .{ }^{22}$

Similarly, let $N$ be the number of distinct elements in $\left\{i_{t}\right\}_{t=0}^{T+1}$ with $2 \leq N \leq(T+2)$. Define a second index $n$ with $n=1,2, \ldots, N$ and the corresponding type sequence $\left\{\widetilde{i}_{n}\right\}_{n=1}^{N}$ with $\widetilde{i}_{n} \in\left\{i_{t}\right\}_{t=0}^{T+1}$ such that $\widetilde{i}_{n}<\widetilde{i}_{n+1}, \forall n<N$. In other words, $n$ is a reordering of distinct elements in $\left\{i_{t}\right\}_{t=0}^{T+1}$ such that $\widetilde{i}_{n}$ is an increasing sequence. Notice that $\widetilde{i}_{1}=0$ and $\widetilde{i}_{N}=1$.

We will construct $N \cdot(T+2)$ points of $\widetilde{\Psi}(i, \omega)$, all collectively denoted by $\left\{\widetilde{a}_{n, t}\right\}_{n=1, t=0}^{N, T+1}$, such that $\widetilde{a}_{n, t}=\widetilde{\Psi}\left(\widetilde{i}_{n}, \omega_{t}\right)$.

Notice first that equilibrium requires that $\widetilde{a}_{n, t}=a_{t}$ if $\widetilde{i}_{n}=i_{t}$. This leaves $(N-1) \cdot(T+$ 2 ) points free for construction. To complete the finite construction, we specify an explicit algorithm to construct a weakly increasing sequence $\left\{\widetilde{a}_{n, t}\right\}_{n=1, t=0}^{N, T+1}$.

Algorithm 1 A recursive algorithm to construct a weakly increasing $\left\{\widetilde{a}_{n, t}\right\}_{n=1, t=0}^{N, T+1}$.

Step 0: Define an initial condition for $t=0$ as $\widetilde{a}_{n, 0}=a_{0}=\min A, \forall 1 \leq n \leq N$.

${ }^{22}$ The specific values of $\omega_{0}$ and $\omega_{T+1}$ are not essential as long as they satisfy $\omega_{0}<\min \Omega$ and $\omega_{T+1}>\max \Omega$. 
Step 1: For observation $t$ with $1 \leq t \leq T$, find $1 \leq n_{t}^{*} \leq N$ such that $\widetilde{i}_{n_{t}^{*}}=i_{t}$. Let $\widetilde{a}_{n_{t}^{*}, t}=a_{t}$. For $1 \leq n \leq N$ and $n \neq n_{t}^{*}$, define $\widetilde{a}_{n, t}$ as an average of two points

$$
\widetilde{a}_{n, t}=\frac{1}{2}\left(\widetilde{a}_{n, t}^{\min }+\widetilde{a}_{n, t}^{\max }\right)
$$

where $\widetilde{a}_{n, t}^{\min }$ and $\widetilde{a}_{n, t}^{\max }$ are defined from $\left\{\widetilde{a}_{n_{t}^{*}, t},\left\{\widetilde{a}_{n, t-1}\right\}_{n=1}^{N}\right\}$ in a recursion starting from $n_{t}^{*}$ as

$$
\widetilde{a}_{n, t}^{\min }=\left\{\begin{array}{cc}
\max \left\{\widetilde{a}_{n-1, t}, \widetilde{a}_{n, t-1}\right\} & \text { if } n>n_{t}^{*} \\
\widetilde{a}_{n, t-1} & \text { if } n<n_{t}^{*}
\end{array}\right.
$$

and

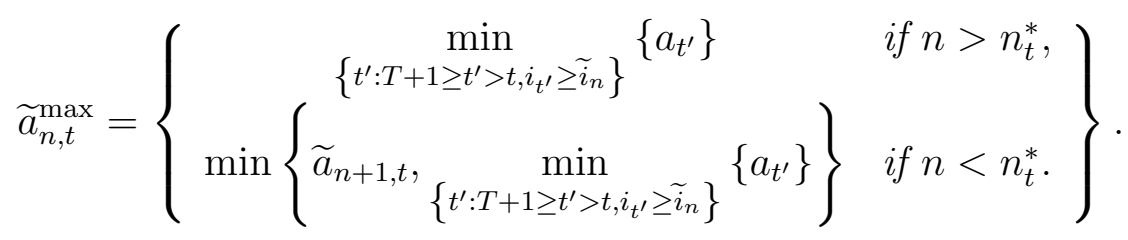

Step 2: If $t<T$, then repeat Step 1 for $t+1$; else go to Step 3.

Step 3: Let $\widetilde{a}_{n, T+1}=a_{T+1}=\max A, \forall 1 \leq n \leq N$ and stop.

For each $1 \leq t \leq T$, the Algorithm starts by producing the realized equilibrium policy outcome, $\widetilde{a}_{n_{t}^{*}, t}=a_{t}$. Starting from $n_{t}^{*}$, the Algorithm then proceeds to a two-way recursion towards both the left and right sides of $n_{t}^{*}$. It is easy to see that the Algorithm produces a non-empty sequence of real numbers. In addition, $\widetilde{a}_{n, t} \in A, \forall n, t$, since every operation involved, including min, max and mean, is a closed operation. Notice that $\widetilde{a}_{n, 0}=\min A$ and $\widetilde{a}_{n, T+1}=\max A$. As a result, we only need to check that $\left\{\widetilde{a}_{n, t}\right\}_{n=1, t=1}^{N, T}$ is a weakly increasing sequence in $(n, t)$.

Verification of Algorithm 1. Start from $t=1$ and we prove the weak monotonicity of $\widetilde{a}_{n, t}$ in $n$. We do this in two steps.

Step 1: $\widetilde{a}_{n, t}^{\max } \geq \widetilde{a}_{n, t} \geq \widetilde{a}_{n, t}^{\min }$ for every $n \neq n_{t}^{*}$. Because $\widetilde{a}_{n, t}=\frac{1}{2}\left(\widetilde{a}_{n, t}^{\min }+\widetilde{a}_{n, t}^{\max }\right)$, it suffices to show that $\widetilde{a}_{n, t}^{\max } \geq \widetilde{a}_{n, t}^{\min }$. We prove the fact for several cases of $n$. First, $\widetilde{a}_{n, t}^{\max } \geq \widetilde{a}_{n, t}^{\min }$ for $1 \leq n<n_{t}^{*}$. From Step 0 of the Algorithm, it follows that $\widetilde{a}_{n, t}^{\max } \geq \min A=\widetilde{a}_{n, t-1}=\widetilde{a}_{n, t}^{\min }$ for every $1 \leq n<n_{t}^{*}$. Second, $\widetilde{a}_{n, t}^{\max } \geq \widetilde{a}_{n, t}^{\min }$ for $n=n_{t}^{*}+1$. Recall that $a_{t^{\prime}} \geq a_{t}$ whenever $t^{\prime}>t$ and $i_{t^{\prime}} \geq i_{t}$. Hence, by taking the minimum we have $\widetilde{a}_{n, t}^{\max } \geq a_{t}$. In addition, $\widetilde{a}_{n, t}^{\max } \geq \widetilde{a}_{n, t-1}=\min A$. For $n=n_{t}^{*}+1$, it follows that $\widetilde{a}_{n, t}^{\max } \geq \max \left\{a_{t}, \widetilde{a}_{n, t-1}\right\}=\max \left\{\widetilde{a}_{n-1, t}, \widetilde{a}_{n, t-1}\right\}=\widetilde{a}_{n, t}^{\min }$. Third, $\widetilde{a}_{n, t}^{\max } \geq \widetilde{a}_{n, t}^{\min }$ for $n_{t}^{*}+1<n \leq N$. For $n=n_{t}^{*}+2$, notice that $\widetilde{a}_{n, t}^{\max } \geq \widetilde{a}_{n-1, t}^{\max } \geq \widetilde{a}_{n-1, t}$, where the first inequality follows because $\widetilde{i}_{n+1}>\widetilde{i}_{n}$ so that the set for min operation in the former is a subset of the latter, and the second inequality from the last result $\widetilde{a}_{n-1, t}^{\max } \geq \widetilde{a}_{n-1, t}^{\min }$ so that $\widetilde{a}_{n-1, t}^{\max } \geq \widetilde{a}_{n-1, t}$ for $n-1=n_{t}^{*}+1$. Using this and the fact that $\widetilde{a}_{n, t}^{\max } \geq \widetilde{a}_{n, t-1}$, the same argument as in the previous step can establish that $\widetilde{a}_{n, t}^{\max } \geq \widetilde{a}_{n, t}^{\min }$ for $n=n_{t}^{*}+2$. By induction, the same inequality holds for every $n_{t}^{*}+1<n \leq N$. 
Step 2: $\widetilde{a}_{n, t}$ is weakly increasing in $n$ for $t=1$. From the construction, $\widetilde{a}_{n, t}^{\min } \geq \widetilde{a}_{n-1, t}$ for $n>n_{t}^{*}$ and $\widetilde{a}_{n, t}^{\max } \leq \widetilde{a}_{n+1, t}$ for $n<n_{t}^{*}$. Since $\widetilde{a}_{n, t}^{\min } \leq \widetilde{a}_{n, t} \leq \widetilde{a}_{n, t}^{\max }$ as shown in Step 1 , we have $\widetilde{a}_{n-1, t} \leq \widetilde{a}_{n, t} \leq \widetilde{a}_{n+1, t}$.

For $1<t \leq T$, the weak monotonicity of $\widetilde{a}_{n, t}$ in $n$ is shown from an induction argument. Specifically, for each $t>1$, we assume that $\widetilde{a}_{n, t-1}^{\max } \geq \widetilde{a}_{n, t-1} \geq \widetilde{a}_{n, t-1}^{\min }$ for every $n \neq n_{t-1}^{*}$, and $\widetilde{a}_{n, t-1}$ is weakly increasing in $n$, as derived for $t=1$. Then we revisit the proof of Step 1 and Step 2 as in $t=1$. It is easy to see that Step 2 is intact, provided that Step 1 holds. For Step 1, a close reading of the proof for $t=1$ reveals that we only need to reestablish that $\widetilde{a}_{n, t}^{\max } \geq \widetilde{a}_{n, t-1}$, which follows from a series of claims. ${ }^{23}$

Claim 1: $\min _{\left\{t^{\prime}: t^{\prime}>t-1, i_{t^{\prime}} \geq \widetilde{i}_{n}\right\}}\left\{a_{t^{\prime}}\right\} \geq \widetilde{a}_{n, t-1}$ for every $1 \leq n \leq N$ and $1<t \leq T$. For $n \neq n_{t-1}^{*}$, $\min _{\left\{t^{\prime}: t^{\prime}>t-1, i_{t^{\prime}} \geq \widetilde{i}_{n}\right\}}\left\{a_{t^{\prime}}\right\} \geq \widetilde{a}_{n, t-1}^{\max } \geq \widetilde{a}_{n, t-1}$, where the first inequality holds by construction, and the second inequality is true from the assumption of induction. For $n=n_{t-1}^{*}$, recall that $a_{t^{\prime}} \geq a_{t-1}$ whenever $t^{\prime}>t-1$ and $i_{t^{\prime}} \geq i_{t-1}=\widetilde{i}_{n}$. Take the minimum to get $\min _{\left\{t^{\prime}: t^{\prime}>t-1, i_{t^{\prime}} \geq \widetilde{i}_{n}\right\}}\left\{a_{t^{\prime}}\right\} \geq a_{t-1}=$ $\widetilde{a}_{n_{t-1}^{*}, t-1}=\widetilde{a}_{n, t-1}$.

Claim 2: $\min _{\left\{t^{\prime}: t^{\prime}>t, i_{t^{\prime}} \geq \widetilde{i}_{n}\right\}}\left\{a_{t^{\prime}}\right\} \geq \widetilde{a}_{n, t-1}$ for every $1 \leq n \leq N$ and $1<t \leq T$. Notice that $\min _{\left\{t^{\prime}: t^{\prime}>t, i_{t^{\prime}} \geq \widetilde{i}_{n}\right\}}\left\{a_{t^{\prime}}\right\} \geq \underset{\left\{t^{\prime}: t^{\prime}>t-1, i_{t^{\prime}} \geq \tilde{i}_{n}\right\}}{\min }\left\{a_{t^{\prime}}\right\}$, since the set for min operation in the former is a subset of the latter. The result then follows from the Claim 1.

Claim 3: $\widetilde{a}_{n_{t}^{*}, t} \geq \widetilde{a}_{n_{t}^{*}, t-1}$ for every $t>1$. Notice that $a_{t} \geq \min _{\left\{t^{\prime}: t^{\prime}>t-1, i_{t^{\prime}} \geq \tilde{i}_{n}\right\}}\left\{a_{t^{\prime}}\right\}$ for $n=n_{t}^{*}$, since $a_{t^{\prime}}$ with $t^{\prime}=t$ and $i_{t}=\widetilde{i}_{n_{t}^{*}}$ is one member of the constraint set. From the Claim 1, we have $\widetilde{a}_{n_{t}^{*}, t}=a_{t} \geq \widetilde{a}_{n_{t}^{*}, t-1}$.

Claim 4: $\widetilde{a}_{n, t}^{\max } \geq \widetilde{a}_{n, t-1}$ for $1 \leq n<n_{t}^{*}$. From the definition of $\widetilde{a}_{n, t}^{\max }$ for $1 \leq n<n_{t}^{*}$ and Claim 2, we only need to prove that $\widetilde{a}_{n+1, t} \geq \widetilde{a}_{n, t-1}$. Furthermore, it suffices to show that $\widetilde{a}_{n+1, t} \geq \widetilde{a}_{n+1, t-1}$, because $\widetilde{a}_{n+1, t-1} \geq \widetilde{a}_{n, t-1}$ from the weak monotonicity assumption of induction for $t-1$. For $n=n_{t}^{*}-1, \widetilde{a}_{n+1, t}=\widetilde{a}_{n_{t}^{*}, t} \geq \widetilde{a}_{n+1, t-1}$ from Claim 3. In addition, by repeating the Step 1 as in $t=1$, we have $\widetilde{a}_{n, t} \geq \widetilde{a}_{n, t}^{\min }=\widetilde{a}_{n, t-1}$ for $n=n_{t}^{*}-1$. But this implies $\widetilde{a}_{n, t}^{\max } \geq \widetilde{a}_{n, t-1}$ for $n=n_{t}^{*}-2$. By induction, the result holds for any $n<n_{t}^{*}$.

Claim 5: $\widetilde{a}_{n, t}^{\max } \geq \widetilde{a}_{n, t-1}$ for $n_{t}^{*}<n \leq N$. This follows immediately from Claim 2.

To summarize, we just proved that the Algorithm produces a weakly increasing sequence in $n$ for each $0 \leq t \leq T+1$. It remains to show that $\widetilde{a}_{n, t}$ is weakly increasing in $t$ for every $1 \leq n \leq N$. From the construction of $\widetilde{a}_{n, t}^{\min }$, for any $t$ and any $n \neq n_{t}^{*}, \widetilde{a}_{n, t} \geq \widetilde{a}_{n, t}^{\min } \geq \widetilde{a}_{n, t-1}$.

\footnotetext{
${ }^{23}$ Recall that $\widetilde{a}_{n, t}^{\max } \geq \widetilde{a}_{n, t-1}$ holds trivially for $t=1$ from Step 0 of the Algorithm, which cannot be taken as given any more for $1<t \leq T$.
} 
For $n=n_{t}^{*}$, from the Claim $3, \widetilde{a}_{n_{t}^{*}, t} \geq \widetilde{a}_{n_{t}^{*}, t-1}$. Consequently, $\widetilde{a}_{n, t} \geq \widetilde{a}_{n, t-1}, \forall t, n$. This finishes the verification of the Algorithm.

Having constructed the points $\left\{\widetilde{a}_{n, t}\right\}_{n=1, t=0}^{N, T+1}$ and corresponding regular grid points $\left(\left\{\widetilde{i}_{n}\right\}_{n=1}^{N},\left\{\omega_{t}\right\}_{t=0}^{T+1}\right)$ from the algorithm, all that remains is to extend the construction to the full function $\widetilde{\Psi}(i, \omega)$. For this purpose, a standard bilinear interpolating spline can be used (for an introduction to splines, see Judd (1998)). Specifically, for each $\left.i \in \widetilde{i}_{n}, \widetilde{i}_{n+1}\right]$ and $\omega \in\left[\omega_{t}, \omega_{t+1}\right]$, a unique bilinear piece can be constructed as

$$
\widetilde{\Psi}(i, \omega)=b_{n, t}^{0}+b_{n, t}^{1} i+b_{n, t}^{2} \omega+b_{n, t}^{3} i \omega,
$$

such that $\widetilde{\Psi}\left(\widetilde{i}_{n}, \omega_{t}\right)=\widetilde{a}_{n, t}, \widetilde{\Psi}\left(\widetilde{i}_{n}, \omega_{t+1}\right)=\widetilde{a}_{n, t+1}, \widetilde{\Psi}\left(\widetilde{i}_{n+1}, \omega_{t}\right)=\widetilde{a}_{n+1, t}$, and $\widetilde{\Psi}\left(\widetilde{i}_{n+1}, \omega_{t+1}\right)=$ $\widetilde{a}_{n+1, t+1}$.

By construction, $\widetilde{\Psi}(i, \omega)$ is continuous in $(i, \omega)$. In addition, a bilinear spline preserves the monotonicity property in each dimension: if $\left\{\widetilde{a}_{n, t}\right\}_{n=1, t=0}^{N, T+1}$ is a weakly increasing sequence in $n$ (resp. $t$ ), then the constructed $\widetilde{\Psi}(i, \omega)$ is weakly increasing in $i$ for each fixed $\omega \in \Omega$ (resp. in $\omega$ for each fixed $i \in[0,1]$ ). Because of the symmetry in $(i, \omega)$, it suffices to show the property for $i$, or $\frac{\partial \widetilde{\Psi}(i, \omega)}{\partial i}=b_{n, t}^{1}+b_{n, t}^{3} \omega \geq 0$. Notice that $\widetilde{\Psi}(i, \omega)$ is linear in $i$ for each fixed $\omega$, in particular for each $\omega_{t}$ and $\omega_{t+1}$. Hence, $\widetilde{a}_{n+1, t} \geq \widetilde{a}_{n, t}$ and $\widetilde{a}_{n+1, t+1} \geq \widetilde{a}_{n, t+1}$ imply that $\frac{\partial \widetilde{\Psi}\left(i, \omega_{t}\right)}{\partial i}=b_{n, t}^{1}+b_{n, t}^{3} \omega_{t} \geq 0$ and $\frac{\partial \widetilde{\Psi}\left(i, \omega_{t+1}\right)}{\partial i}=b_{n, t}^{1}+b_{n, t}^{3} \omega_{t+1} \geq 0$. It immediately follows that $\frac{\partial \widetilde{\Psi}(i, \omega)}{\partial i}=b_{n, t}^{1}+b_{n, t}^{3} \omega \geq 0$ for every $\omega \in\left[\omega_{t}, \omega_{t+1}\right]$.

With the extension to the full function $\widetilde{\Psi}$, the proof of Theorem 4 is complete.

\section{Proof of Theorem 5.}

Sufficiency. Fix any policy data and weight $\alpha$ that satisfy the implication in (13). Consider any $u$ of the form

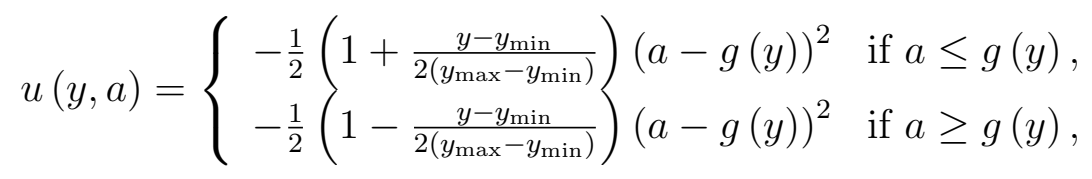

where $y_{\min }=\min _{i \in[0,1], \omega \in \Omega} y(i, \omega), y_{\max }=\max _{i \in[0,1], \omega \in \Omega} y(i, \omega)$, and $g(y)$ is continuous and weakly increasing. It is clear that $u(y, a)$ is continuous in $y$ and single-peaked in $a$. Following the same argument as in the counterpart of the proof of Theorem $4, u(y, a)$ satisfies strict singlecrossing in $(a ; y)$. As a result, any $U(i, \omega, a)$ defined by $U(i, \omega, a)=u(y(i, \omega), a)$ satisfies (A1), (A2) and (A4).

For a given $\alpha$, define the income of the pivotal decision maker in $t$ as $y_{t}=y\left(\mu\left(\omega_{t}, \alpha\right), \omega_{t}\right)$. To prove the result, it suffices to show that there exists a $g(y)$ such that $a_{t}=g\left(y_{t}\right)$. Because 
$a_{t}<a_{\tau} \Longrightarrow y_{t}<y_{\tau}$, we have $y_{t}=y_{\tau} \Longrightarrow a_{t}=a_{\tau}$. Hence, by redefining and reordering $t$ if necessary, without loss of generality we can assume that $\left\{y_{t}\right\}_{t=1}^{T}$ is an increasing sequence in $t$, and $\left\{a_{t}\right\}_{t=1}^{T}$ is weakly increasing. For any $1 \leq t \leq T-1$ and $y \in\left[y_{t}, y_{t+1}\right]$, define

$$
g(y)=a_{t}+\frac{a_{t+1}-a_{t}}{y_{t+1}-y_{t}}\left(y-y_{t}\right)
$$

and

$$
\begin{aligned}
& g(y)=\min A+\frac{a_{1}-\min A}{y_{1}} y, \forall y \leq y_{1}, \\
& g(y)=a_{T}+\frac{\max A-a_{T}}{\max _{\omega \in \Omega}\{y(1, \omega)\}-y_{T}}\left(y-y_{T}\right), \forall y \geq y_{T} .
\end{aligned}
$$

It is clear that $g(y)$ is weakly increasing and $a_{t}=g\left(y_{t}\right)$. This finishes the proof.

Proof of Proposition 2 We prove the result by showing that neither candidate has an incentive to deviate from the proposed equilibrium. Given the specified tie-breaking rule, i.e., ties default to $a^{1}$, candidate 1 wins the election if $a^{1}=a^{2}$, hence has no incentive to deviate. Now consider a policy deviation by candidate 2, and without loss of generality, let $a^{2}>a^{1}=\arg \max _{a} U(\mu(\omega, \alpha), \omega, a)$.

To analyze the consequence of policy deviation on the campaign contribution and voting, we need to specify a rational belief on the winning policy between $a^{1}$ and $a^{2}$. We conjecture and later verify one consistent belief under which $a^{1}$ is the winning policy against the proposed deviation. Based on this belief, each type $i$ contributes an amount $z_{i}^{*}=z^{*}\left(y(i, \omega), a^{1}\right)$, and supports the policy platform $a^{j}$ if $V\left(i, \omega, a^{j}, z_{i}^{*}\right)>V\left(i, \omega, a^{-j}, z_{i}^{*}\right)$. Denote $x$ as the type who is indifferent between $a^{1}$ and $a^{2}$, i.e., $V\left(x, \omega, a^{1}, z_{x}^{*}\right)=V\left(x, \omega, a^{2}, z_{x}^{*}\right)$. To verify the belief, it suffices to show that $x>\mu(\omega, \alpha)$, so that under single crossing property of $V$, $Z^{1}(\omega ; \alpha)>Z^{2}(\omega ; \alpha)$ and $\gamma\left(Z^{1}\right)>\gamma\left(Z^{2}\right)$, i.e., candidate 1 wins the election. But $x>\mu(\omega, \alpha)$ holds by construction. To see this, note that by definition, $a^{1}$ leads to the maximum utility $U$ for $\mu(\omega, \alpha)$ when he can choose $a$ and $z$ at the same time. In particular, the maximum utility for $\mu(\omega, \alpha)$ is equal to $U\left(\mu(\omega, \alpha), \omega, a^{1}\right)$, or equivalently $V\left(\mu(\omega, \alpha), \omega, a^{1}, z_{\mu(\omega, \alpha)}^{*}\right)$. It then follows that $V\left(\mu(\omega, \alpha), \omega, a^{1}, z_{\mu(\omega, \alpha)}^{*}\right)>V\left(\mu(\omega, \alpha), \omega, a^{2}, z_{\mu(\omega, \alpha)}^{*}\right)$, hence $x>\mu(\omega, \alpha)$ by the single crossing property of $V$. This verifies the belief that $a^{1}$ beats $a^{2}$. Consequently, candidate 2 has no incentive to deviate from $a^{2}=a^{1}$.

\section{References}

[1] Acemoglu, D. and J. Robinson (2008), "Persistence of Powers, Elites and Institutions," American Economic Review, 98: 267-293.

[2] Afriat, S. (1967), "The Construction of a Utility Function from Expenditure Data," International Economic Review, 8: 67-77. 
[3] Austen-Smith, D. (1987), "Interest Groups, Campaign Contributions, and Probabilistic Voting", Public Choice 54: 123-139.

[4] Baron, D. (1994), "Electoral Competition with Informed and Uniformed Voters," American Political Science Review, 88: 33-47.

[5] Bartels, L. (2008), Unequal Democracy: The Political Economy of the New Gilded Age, Princeton, NJ: Princeton University Press.

[6] Bénabou, R. (1996), "Inequality and Growth,"NBER Macroeconomics Annual, B. Bernanke and J. Rotemberg, eds., 11-74.

[7] Bénabou, R. (2000), "Unequal Societies: Income Distribution and the Social Contract," American Economic Review, 90(1): 96-129.

[8] Boldrin, M. and L. Montruchio (1986), "On the Indeterminacy of Capital Accumulation Paths," Journal of Economic Theory, 40: 26-39.

[9] Bourguignon, F., and T. Verdier (2000), "Oligarchy, Democracy, Inequality and Growth," Journal of Development Economics, 62: 287-313.

[10] Brown, D. and F. Kubler (2008), Computational Aspects of General Equilibrium Theory: Refutable Theories of Value, Springer-Verlag.

[11] Campante, F. (2011), "Redistribution in a Model of Voting and Campaign Contributions," Journal of Public Economics, 95: 646-56.

[12] Chiappori, P.-A. and J.-C. Rochet (1987), "Revealed Preference and Differentiable Demand, Econometrica, 55: 687-91.

[13] Coate, S. (2004), "Political Competition with Campaign Contributions and Informative Advertising", Journal of the European Economic Association, 2: 772-804.

[14] Debreu, G. (1974). "Excess Demand Functions," Journal of Mathematical Economics, 1: $15-21$.

[15] Degan, A. and A. Merlo (2009), “ Do Voters Vote Ideologically?” Journal of Economic Theory, 144:1868-94.

[16] Feddersen T. and A. Sandroni (2006), "A Theory of Participation in Elections," American Economic Review, 96: 1271-1282.

[17] Gallop Poll Social Series: Economy and Personal Finance (2011), Timberline: 927910 G: 745, Princeton Job \#: 11-04-006, conducted on April 7-11, 2011 by Jeff Jones and Lydia Saad.

[18] Gans, J. and M. Smart (1996), "Majority voting with single-crossing preferences," Journal of Public Economics, 59: 219-237.

[19] Gilens, M. (2005), "Inequality and Democratic Responsiveness," Public Opinion Quarterly 69 (5): 778-896.

[20] Grossman, G. and E. Helpman (1996), "Electoral Competition and Special Interest Policies", Review of Economic Studies, 63: 265-286. 
[21] Jacobs, L.R. and B.I. Page (2005), "Who Influences U.S. Foreign Policy?" American Political Science Review, 99: 107-124.

[22] Judd, K. (1998), Numerical Methods in Economics, Cambridge: MIT Press.

[23] Kalandrakis, T. (2010), "Rationalizable Voting," Theoretical Economics, 5:93-125.

[24] Lindbeck, A. and J. Weibull (1993), "A model of political equilibrium in a representative democracy," Journal of Public Economics, 51(2: 195-209.

[25] Mantel, R. (1974), "On the characterization of aggregate excess demand," Journal of Economic Theory 7: 348-353.

[26] Manza, J. and F.L. Cook (2002), "A Democratic Polity? Three Views of Policy Responsiveness to Public Opinion in the United States." American Political Research, 30: 630-67.

[27] Prat, A. (2002), "Campaign Spending with Office-Seeking Politicians, Rational Voters, and Multiple Lobbies", Journal of Economic Theory, 103: 162-189.

[28] Richter, M. K. (1966), "Revealed Preference Theory," Econometrica, 34: 635 - 645.

[29] Riker, W. and P. Ordeshook (1968), "A Theory of the Calculus of Voting," American Political Science Review, 62(1): 25-42.

[30] Rosenstone, S. and J.M. Hansen (1993), Mobilization, Participation and Democracy in America, Macmillan, New York.

[31] Rothstein, P. (1990), "Order Restricted Preferences and Majority Rule," Social Choice and Welfare, 7: 331-42.

[32] Sonnenschein, H. (1973), "Do Walras' Identity and Continuity Characterize the Class of Community Excess Demand Functions?", Journal of Economic Theory 6: 345-354.

[33] USA Today/Gallop Poll (2010), Timberline: 927592 G: 593, Princeton Job \#: 10-11-021 conducted November 19-21, 2010 by Jeff Jones and Lydia Saad.

[34] Varian, H. (1982), "The Non-Parametric Approach to Demand Analysis," Econometrica, 50: $945-974$.

[35] Varian, H. (2006), "Revealed Preference," In: Michael Szenberg, Lall Ramrattan, Aron A. Gottesman (Eds.), Samuelsonian Economics and the Twenty-First Century, Oxford University Press, pp. 99-115. 